\title{
TWO NEW LONG-PERIOD GIANT PLANETS FROM THE MCDONALD OBSERVATORY PLANET SEARCH AND TWO STARS WITH LONG-PERIOD RADIAL VELOCITY SIGNALS RELATED TO STELLAR ACTIVITY CYCLES
}

\author{
Michael Endl ${ }^{1}$, Erik J. Brugamyer ${ }^{1}$, William D. Cochran ${ }^{1}$, Phillip J. MacQueen ${ }^{1}$, Paul Robertson ${ }^{1,12,13}$, \\ Stefano Meschiari ${ }^{1}$, Ivan Ramirez ${ }^{1}$, Matthew Shetrone ${ }^{1}$, Kevin Gullikson ${ }^{1}$, Marshall C. Johnson ${ }^{1}$, \\ Robert Wittenmyer ${ }^{2}$, Jonathan Horner ${ }^{3,4}$, David R. Ciardi ${ }^{5}$, Elliott Horch ${ }^{6}$, Attila E. Simon ${ }^{7}$, \\ ${\text { Steve B. } \text { Howell }^{8} \text {, Mark Everett }}^{9}$, Caroline Caldwell $^{10}$, and Barbara G. Castanheira ${ }^{11}$ \\ ${ }^{1}$ McDonald Observatory and Department of Astronomy, University of Texas at Austin, Austin, TX 78712, USA; mike@astro.as.utexas.edu \\ ${ }^{2}$ School of Physics and Australian Centre for Astrobiology, UNSW Australia, Sydney, NSW 2052, Australia \\ ${ }^{3}$ Computational Engineering and Science Research Centre, University of Southern Queensland, Toowoomba, Queensland 4350, Australia \\ ${ }^{4}$ Australian Centre for Astrobiology, UNSW Australia, Sydney, NSW 2052, Australia
${ }^{5}$ NASA Exoplanet Science Institute \& Infrared Processing and Analysis Center, California Institute of Technology, Pasadena, CA 91125, USA \\ ${ }^{6}$ Department of Physics, Southern Connecticut State University, 501 Crescent St., New Haven, CT 06515, USA \\ ${ }^{7}$ Physikalisches Institut, Center for Space and Habitability, University of Bern, CH-3012 Bern, Switzerland \\ ${ }^{8}$ NASA Ames Research Center, Moffett Field, CA 94035, USA \\ ${ }^{9}$ National Optical Astronomy Observatory, 950 North Cherry Avenue, Tucson, AZ 85719, USA \\ ${ }^{10}$ Astrophysics Research Institute, Liverpool John Moores University, 146 Brownlow Hill, Liverpool, L3 5RF, UK \\ ${ }^{11}$ McDonald Observatory, University of Texas at Austin, Austin, TX 78712, USA \\ Received 2015 October 2; accepted 2015 December 7; published 2016 February 4
}

\begin{abstract}
We report the detection of two new long-period giant planets orbiting the stars HD 95872 and HD $162004\left(\psi^{1}\right.$ Dra B) by the McDonald Observatory planet search. The planet HD 95872b has a minimum mass of $4.6 M_{\text {Jup }}$ and an orbital semimajor axis of 5.2 AU. The giant planet $\psi^{1} \mathrm{Dra} \mathrm{Bb}$ has a minimum mass of $1.5 M_{\text {Jup }}$ and an orbital semimajor axis of 4.4 AU. Both of these planets qualify as Jupiter analogs. These results are based on over one and a half decades of precise radial velocity (RV) measurements collected by our program using the McDonald Observatory Tull Coude spectrograph at the $2.7 \mathrm{~m}$ Harlan J. Smith Telescope. In the case of $\psi^{1}$ Dra B we also detect a long-term nonlinear trend in our data that indicates the presence of an additional giant planet, similar to the Jupiter-Saturn pair. The primary of the binary star system, $\psi^{1}$ Dra A, exhibits a very large amplitude RV variation due to another stellar companion. We detect this additional member using speckle imaging. We also report two cases_-HD 10086 and HD 102870 ( $\beta$ Virginis) — of significant RV variation consistent with the presence of a planet, but that are probably caused by stellar activity, rather than reflexive Keplerian motion. These two cases stress the importance of monitoring the magnetic activity level of a target star, as long-term activity cycles can mimic the presence of a Jupiter-analog planet.
\end{abstract}

Key words: planetary systems - stars: activity - techniques: radial velocities

Supporting material: machine-readable tables

\section{INTRODUCTION}

"How common are solar system analogs?" Until relatively recently, this fundamental question had little in the way of observational answers. Although the Kepler mission (Borucki et al. 2010) has provided first constraints on the answer to the related question "How common are Earth analogs?" until our instruments and techniques improve to the point that we are capable of detecting planets across a range of masses and orbits analogous to those of the planets in our solar system, a definitive answer will be beyond our reach. However, as a next step we might instead ask, "How common are Jupiter analogs?"- gas giant planets that have either not significantly migrated inward from the location of their formation beyond the ice line in the protoplanetary disk or migrated inward very early, followed by an episode of outward migration (the "Grand Tack" model; Walsh et al. 2011). As the time baseline of radial velocity (RV) searches grows, we are becoming better equipped to answer this last question.

\footnotetext{
12 Center for Exoplanets and Habitable Worlds, Department of Astronomy and Astrophysics, The Pennsylvania State University.

${ }^{13}$ NASA Sagan Fellow.
}

The RV technique has been used to detect/discover $~ 600$ of the 2000 known, confirmed exoplanets. Since the technique is heavily biased toward massive planets in short-period orbits, the majority of these are gas giants in orbits of less than one Earth year. Only about $25 \mathrm{RV}$-detected planets can be considered "Jupiter analogs," which we define as within a factor of a few Jupiter masses and in orbits longer than 8 yr (about 3000 days). Although the Kepler mission-utilizing the planet transit method-has delivered $\sim 1000$ planets and nearly 5000 candidates, none of these can be classified as "long-period," owing to the limited time baseline of the mission data.

To answer the question of the uniqueness of our solar system, it is probably more important to find and characterize long-period Jovian planets than to find small-radius terrestrial planets. Other studies (Wittenmyer et al. 2011; Howard et al. 2012; Fressin et al. 2013; Petigura et al. 2013) have shown that terrestrial-size planets are quite common around other stars, but the data concerning Jupiter analogs are quite incomplete owing to the need for a time baseline of over 10-15 yr. A handful of RV surveys have "outgrown" this time baseline selection bias: the Lick Observatory planet search from 1987 to 
2011 (Fischer et al. 2014), our ongoing McDonald Observatory Planet Search, the Anglo-Australian Planet Search (e.g., Wittenmyer et al. 2014a), the Keck/HIRES RV survey (e.g., Howard et al. 2014), and the planet search programs at CORALIE (e.g., Marmier et al. 2013) and HARPS (e.g., Moutou et al. 2015). An example of a Jupiter-analog planet orbiting a solar twin is presented in Bedell et al. (2015). While the Kepler mission has revolutionized exoplanetary science and provided a first estimate of the frequency of Earth-size planets in Earth-like orbits, long-term RV surveys complement these data with measurements of the frequency of Jupiter-like planets in Jupiter-like orbits. This in turn will reveal how common solar-system-like architectures are.

While the idea that Jupiter analogs are required to shield terrestrial planets from impacts has been conclusively dismantled (e.g., Horner \& Jones 2008, 2012; Horner et al. 2010), the presence of Jupiter analogs might be critical for the delivery of water to planets that would otherwise have formed as dry, lifeless husks (Raymond 2006; Horner \& Jones 2010). The early dynamical evolution of Jupiter and Saturn might also be responsible for a depletion of the inner planetesemial disk and for the subsequent formation of small, low-mass terrestrial planets, instead of large, massive super-Earths (Batygin \& Laughlin 2015). The search for Jupiter analogs thus provides a key datum for models of planetary formation and evolutionattempting to answer the question "How common are planetary systems like our own?"

The McDonald Observatory Planet Search (Cochran \& Hatzes 1993) is a high-precision RV survey of hundreds of FGKM stars, begun in 1987 using the $2.7 \mathrm{~m}$ Harlan J. Smith Telescope (HJST). Since our migration to our current instrumental configuration in 1998 ("Phase III," described in Hatzes et al. 2003), we achieve routine long-term Doppler velocity precision of $\sim 4-8 \mathrm{~m} \mathrm{~s}^{-1}$. With this precision and an observational time baseline approaching $17 \mathrm{yr}$, we are now sensitive to Jovian analogs. In this paper, we present two new long-period planetary companions (HD 95872b and $\psi^{1}$ Dra $\mathrm{Bb}$ ). We also report two cases (HD 10086 and $\beta$ Virginis) of Keplerian-like signals that mimic a Jupiter-type planet but are probably the result of stellar activity akin to the $11 \mathrm{yr}$ solar cycle.

\section{OBSERVATIONS}

Our RV measurements were obtained using the $2.7 \mathrm{~m} \mathrm{HJST}$ and $10 \mathrm{~m}$ Keck I telescope. The specific instruments/observations are described below.

\subsection{Harlan J. Smith Telescope Observations}

For the $2.7 \mathrm{~m} \mathrm{HJST}$, we utilize the cross-dispersed Echelle Tull Coude spectrograph (Tull et al. 1995). Our configuration uses a 1"!2 slit, an Echelle grating with 52.67 grooves $\mathrm{mm}^{-1}$, and a $2048 \times 2048$ Tektronix CCD with $24 \mu \mathrm{m}$ pixels, yielding a resolving power $(R=\lambda / \Delta \lambda)$ of $R=60,000$. The wavelength coverage extends from 3750 to $10200 \AA$ and is complete from the blue end to $5691 \AA$, after which there are increasingly large interorder gaps.

\subsection{Keck Telescope Observations}

For HD 95872, we also obtained 10 precise RV measurements using Keck I and its HIRES spectrograph (Vogt et al. 1994), during three observing runs allocated to the
NASA CoRoT key science project, during times when the CoRoT fields were unobservable.

The spectra for HD 95872 were taken with HIRES with a resolving power of $R=50,000$, using an instrumental setup similar to the California Planet Search (e.g., Howard et al. 2010). Also for HIRES we used an iodine cell to monitor real-time instrumental variations relevant to measuring precise RVs.

\subsection{Data Reduction}

The raw CCD data were reduced using a pipeline implemented in the Image Reduction and Analysis Facility (IRAF) using standard routines within the echel le package. The process includes overscan trimming, bad pixel processing, bias frame subtraction, scattered light removal, flat-field division, order extraction, and wavelength solution application using a Th-Ar calibration lamp spectrum. Most cosmic rays are successfully removed via IRAF's interpolation routines; however, particularly troublesome hits are removed by hand.

\section{ANALYSIS}

\subsection{RV Measurements}

Our RV measurements were obtained using our standard iodine cell RV reduction pipeline Austral (Endl et al. 2000). Our approach follows the standard iodine cell data analysis methodology: the stellar RV is calculated by comparing all spectra of the target star, taken with the iodine cell, with a high signal-to-noise ratio $(\mathrm{S} / \mathrm{N})$ stellar template spectrum free of iodine lines. During regular RV observations, the temperaturecontrolled iodine cell is inserted in the light path and superimposes a dense reference spectrum onto the stellar spectrum. The iodine lines thus provide a simultaneous wavelength calibration and allow the reconstruction of the shape of the instrumental profile at the time of observation. The iodine cell at the Tull spectrograph has been in regular operation for more than two decades.

\subsection{Stellar Activity Indicators}

As a check against photospheric activity masquerading as planet-like Keplerian motion, we measure the $\mathrm{Ca} \mathrm{H}$ \& $\mathrm{K}$ Mount Wilson $S_{\mathrm{HK}}$ index (Soderblom et al. 1991; Baliunas et al. 1995; Paulson et al. 2002) simultaneously with each RV data point. In addition, we have calculated the line bisector velocity spans (BVSs; e.g., Hatzes et al. 1997) of lines outside the region of iodine cell absorption. These time-series measurements are then checked for any possible correlation (s) with the RV measurements.

\subsection{Stellar Characterization}

We determined stellar atmospheric parameters for all four stars using a traditional absorption line curve-of-growth approach, following a procedure similar to that outlined in Brugamyer et al. (2011). The method utilizes an updated list of suitable Fe and Ti lines, the local thermodynamic equilibrium (LTE) line analysis and spectral synthesis code MOOG, ${ }^{13}$ and a grid of 1D, plane-parallel ATLAS9 (Kurucz 1993) model atmospheres. We first manually measured the equivalent widths

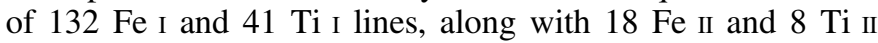

\footnotetext{
13 Available at http://www.as.utexas.edu/ chris/moog.html.
} 
Table 1

Stellar Properties

\begin{tabular}{|c|c|c|c|c|c|c|c|c|c|c|c|}
\hline Star & $\begin{array}{l}\text { Spectral } \\
\text { Type }\end{array}$ & V & $B-V$ & $M_{V}$ & $\begin{array}{l}\text { Parallax } \\
\text { (mas) }\end{array}$ & $\begin{array}{l}\text { Dist. } \\
(\mathrm{pc})\end{array}$ & $\begin{array}{l}T_{\text {eff }} \\
(\mathrm{K})\end{array}$ & $\log g$ & {$[\mathrm{Fe} / \mathrm{H}]$} & $\begin{array}{l}\text { Mass } \\
\left(M_{\odot}\right)\end{array}$ & $\begin{array}{l}\text { Age } \\
(\mathrm{Gyr})\end{array}$ \\
\hline HD 95872 & $\mathrm{~K} 0 \mathrm{~V}$ & 9.895 & 0.827 & 10.50 & 132.30 & 7.56 & $5312 \pm 100$ & $4.43 \pm 0.15$ & $0.41 \pm 0.09$ & $0.95 \pm 0.04$ & $10.0 \pm 3.7$ \\
\hline$\psi^{1}$ Dra B & G0V & 5.699 & 0.562 & 3.97 & 45.13 & 22.16 & $6212 \pm 75$ & $4.20 \pm 0.12$ & $0.01 \pm 0.06$ & $1.19 \pm 0.07$ & $3.3 \pm 1.0$ \\
\hline HD 10086 & G5IV & 6.610 & 0.688 & 4.97 & 46.99 & 21.28 & $5722 \pm 65$ & $4.43 \pm 0.10$ & $0.10 \pm 0.04$ & $1.01 \pm 0.03$ & $5.5 \pm 2.3$ \\
\hline$\beta$ Vir & F8V & 3.589 & 0.568 & 3.40 & 91.65 & 10.91 & $6145 \pm 75$ & $3.98 \pm 0.12$ & $0.15 \pm 0.05$ & $1.34 \pm 0.10$ & $3.2 \pm 0.7$ \\
\hline
\end{tabular}

lines, in our template spectra (without the reference iodine cell in the light path). With these measurements in hand, the stellar effective temperature is constrained by assuming and enforcing excitation equilibrium - by varying the model atmosphere temperature until any trends in derived abundances with temperature are removed. Surface gravity is constrained by assuming and enforcing ionization equilibrium - by varying the model atmosphere gravity until the derived abundances of neutrals and ions agree. Microturbulent velocity is constrained by forcing the derived abundances for stronger lines to match those for weaker lines. For these processes, we used a weighted average of $\mathrm{Fe}(2 \times)$ and $\mathrm{Ti}(1 \times)$ when computing the relevant slopes/offsets (as there are approximately twice as many Fe than $\mathrm{Ti}$ lines). This process is repeated iteratively until all conditions are satisfied simultaneously with a self-consistent set of stellar parameters.

The results of our stellar characterization are summarized in Table 1. Spectral types, photometric data, and parallaxes are taken from the ASCC-2.5 catalog (Version 3; Kharchenko \& Roeser 2009). We also include mass and age estimates from Yonsei-Yale model isochrones (Yi et al. 2001; Kim et al. 2002).

Using the stellar parameters $T_{\text {eff }}, \log g,[\mathrm{Fe} / \mathrm{H}]$, and their errors, we determined the masses and ages of our stars using the procedure outlined in Ramírez et al. (2014, their Section 4.5). Briefly, the location of each star on stellar parameter space was compared with that of stellar interior and evolutionary model predictions. The Yonsei-Yale isochrone grid was used in our implementation. Each isochrone point was given a probability of representing an observation based on its distance from the measured stellar parameters and weighted by the observational errors. Then, mass and age probability distribution functions were computed by adding the probabilities of individual isochrone points binned in mass and age, respectively. The peaks of these distributions were adopted as the most probable mass and age, while the $1 \sigma$-like widths were used to estimate the errors.

Contrary to a more common practice, we did not use parallaxes in our mass and age determinations. This is because one of our stars, HD 95872, does not have a reliable measurement of trigonometric parallax; this star was not included in the Hipparcos catalog. To maintain consistency in our analysis, we employed the spectroscopic $\log g$ values as luminosity indicators instead of absolute magnitudes computed using measured parallaxes. If we had used the Hipparcos parallaxes for the three stars that have those values available, their masses would be only about $0.01 \pm 0.01 M_{\odot}$ smaller.

\footnotetext{
${ }^{14}$ Available at http://www.stefanom.org/systemic/.
}

\subsection{Planetary Orbit Modeling}

We performed our planetary orbit modeling using the Systemic Console ${ }^{14}$ package (Meschiari et al. 2009), a software application for the analysis and fitting of Doppler RV data sets.

\section{THE PLANET AROUND HD 95872}

The star HD 95872 was originally selected for RV monitoring from a sample of 22 thin-disk stars observed on the $2.7 \mathrm{~m} \mathrm{HJST}$ in 1998 for a project to characterize the metalrich end of chemical evolution of the Galactic disk. The sample of 22 stars was selected by M. Grenon (Observatorie de Geneve) for Sandra Castro (ESO) and Matthew Shetrone on the basis of their extreme kinematic (perigalactica $\sim 3 \mathrm{kpc}$ ) and photometric properties.

\subsection{Keplerian Solution}

Table 2 presents the complete set of our RV measurements for HD 95872 from the HJST/Tull survey, as well as 10 additional measurements obtained with Keck/HIRES. The RV coverage spans approximately $11 \mathrm{yr}$ of monitoring over 44 measurements. The median internal uncertainty for our observations is $\approx 6 \mathrm{~m} \mathrm{~s}^{-1}$, and the peak-to-peak velocity is $\approx 137 \mathrm{~m} \mathrm{~s}^{-1}$. The velocity scatter around the average $\mathrm{RV}$ is $\approx 32.1 \mathrm{~m} \mathrm{~s}^{-1}$.

The top panel of Figure 1 shows the error-weighted, normalized Lomb-Scargle periodogram (Zechmeister \& Kürster 2009). The three horizontal lines in the plot represent different levels of false-alarm probability (FAP; 10\%, 1\%, and $0.1 \%$, respectively). The FAPs were computed by scrambling the data set 100,000 times, in order to determine the probability that the power at each frequency could be exceeded by chance (e.g., Kürster et al. 1997; Marcy et al. 2005). Computing the FAPs for this sparse data set required scanning only frequencies that were effectively sampled by the set of observation times. We determined an "effective" Nyquist frequency for the data set using the calculation formula of Koen (2006). For irregularly spaced data sets, the effective Nyquist frequency is much higher than the corresponding Nyquist frequency of a regularly spaced data set of the same size. The algorithm of Koen (2006) finds a clear minimum at $P \approx 2$ days (bottom panel of Figure 1), corresponding to the effective Nyquist frequency for the data. Accordingly, we exclude periods shorter than 2 days from our calculations.

Visual inspection of the 44 individual RV measurements suggests the presence of a sparsely sampled, long-period signal (see top panel of Figure 2). The Lomb-Scargle periodogram (Figure 1) bears this out. The two strongest signals, at $P=29.6$ days $\left(\right.$ FAP $\left.<4.0 \times 10^{-5}\right)$ and $P=331.2$ days $\left(\mathrm{FAP}=1.1 \times 10^{-2}\right)$, have significant power in the window 
Table 2

Differential Radial Velocity Observations for HD 95872 (Sample)

\begin{tabular}{lcrc}
\hline \hline Spectrum & BJD & $\begin{array}{c}\mathrm{dRV} \\
\left(\mathrm{m} \mathrm{s}^{-1}\right)\end{array}$ & $\begin{array}{c}\text { Uncertainty } \\
\left(\mathrm{m} \mathrm{s}^{-1}\right)\end{array}$ \\
\hline 1 & $2,453,073.8686$ & 66.2 & 5.3 \\
2 & $2,453,463.7752$ & 98.5 & 5.9 \\
3 & $2,453,843.7736$ & 109.2 & 5.9 \\
4 & $2,454,557.7575$ & 63.5 & 6.7 \\
5 & $2,455,286.7123$ & -22.2 & 4.1 \\
$6^{\mathrm{a}}$ & $2,455,366.7841$ & 15.5 & 1.9 \\
$7^{\mathrm{a}}$ & $2,455,368.7876$ & 13.3 & 3.5 \\
8 & $\cdots$ & $\cdots$ & $\cdots$ \\
\hline
\end{tabular}

Note.

${ }^{a}$ Observed with Keck/HIRES; all others with the HJST/Tull.

(This table is available in its entirety in machine-readable form.)


Figure 1. Top panel: generalized Lomb-Scargle periodogram for the HD 95872 RV data. FAP levels are shown at the 10\%, 1\%, and $0.1 \%$ level. Middle panel: periodogram of the window function. Bottom panel: determination of the "effective" Nyquist frequency for the data set. Both the effective Nyquist frequency and the corresponding Nyquist frequency for a regularly spaced data set are marked.
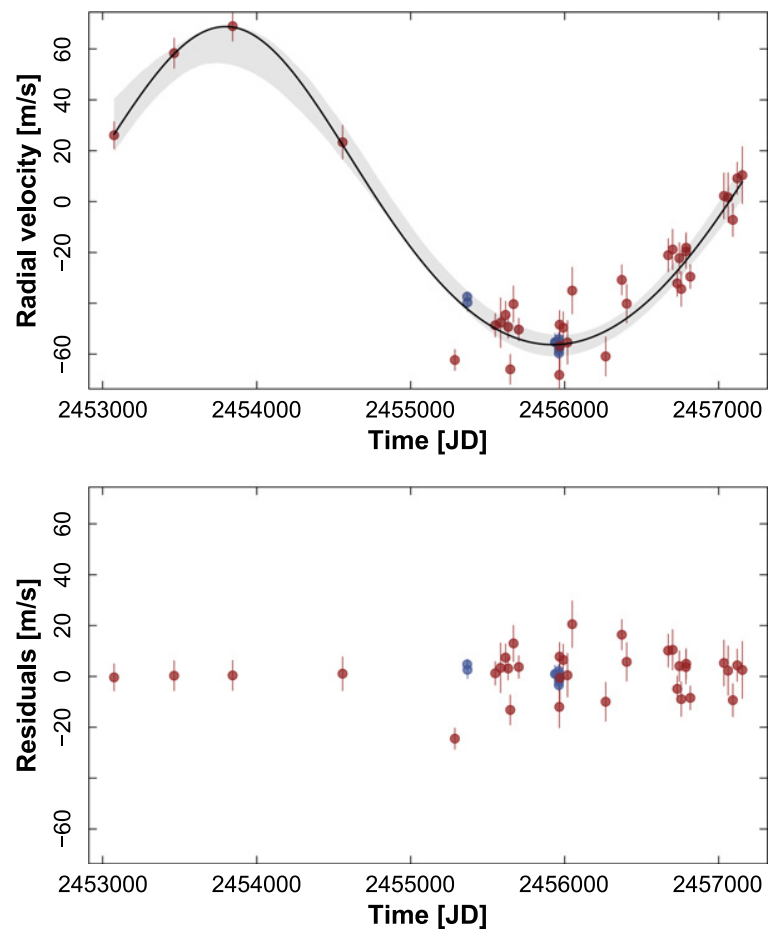

Figure 2. Top panel: our RV data from HJST/Tull (red) and Keck/HIRES (blue) and the best-fit Keplerian model. The shaded area marks the 10\%-90\% percentiles of the RV curves sampled from the MCMC trials and indicates the range of the models compatible with the data. Bottom panel: RV residuals.

function, and they are likely related to the periodicities in the observational cadence (the lunar synodic month and the solar year). The remaining peak is at $P=3922.05$ days $\left(\mathrm{FAP}=2 \times 10^{-4}\right)$. This signal is well fit with a Keplerian orbit of period $P=4278 \pm 169$ days and semiamplitude $K=59 \pm 4 \mathrm{~m} \mathrm{~s}^{-1}$ (Figure 2). Together with the assumed stellar mass of $0.95 \mathcal{M}_{\odot}$, this implies a minimum mass of $\mathcal{M} \sin i=4.6 \pm 0.3 \mathcal{M}_{J}$ and a semimajor axis $a=5.2 \pm 0.13 \mathrm{AU}$. The best-fit orbit for the planet shows a small amount of eccentricity $(e=0.06 \pm 0.04$, broadly consistent with circular). Orbital uncertainties were derived by running a Markov Chain Monte Carlo (MCMC) algorithm (Ford 2005, 2006; Meschiari et al. 2009; Gregory 2011) on the data set. Noninformative priors were adopted over all parameters (uniform in logarithm for mass and period). Marginal distributions of the parameters are shown in Figure 3; no significant correlations among parameters were observed. A summary of the astrocentric orbital elements of HD $95872 b$ is reported in Table 3.

The one-planet fit reduces the rms of the data from 46.8 to $8.1 \mathrm{~m} \mathrm{~s}^{-1}$. The stellar jitter for HD 95872 (that is, the amount of noise added in quadrature to the formal uncertainties required in order to completely fit the residuals) is $8 \pm 2 \mathrm{~m} \mathrm{~s}^{-1}$ and is derived self-consistently from the MCMC analysis. We note that the normalized residuals are very nearly normally distributed, aside from a single outlier (Figure 4).

Figure 5 shows the Lomb-Scargle periodogram of the RV residuals from the one-planet best fit. There is no strong periodicity $(\mathrm{FAP}<10 \%$ ) in the residuals supporting the presence of additional planets in the system. 

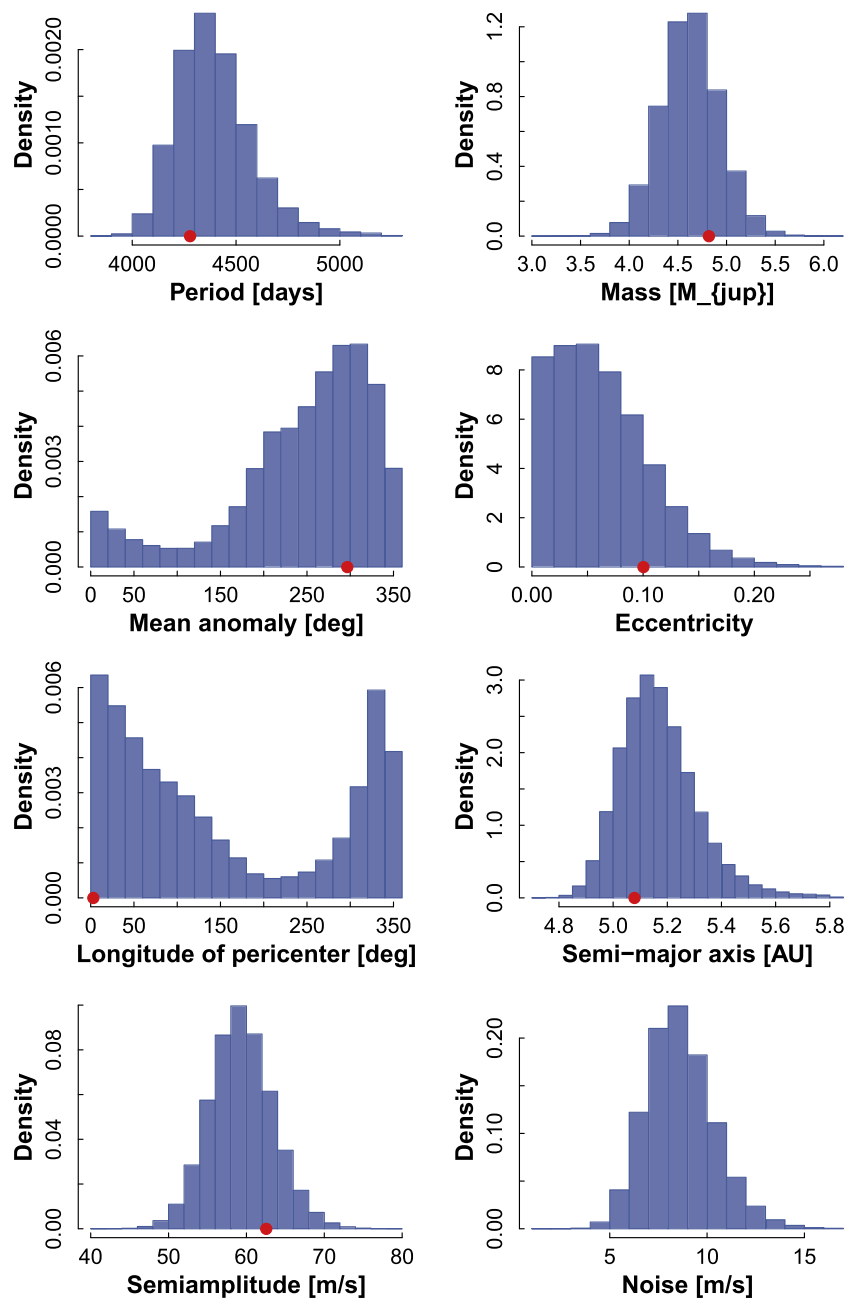

Figure 3. Marginal distributions of the orbital elements of HD 95872b, as computed by the MCMC algorithm. The red dot marks the value of the best-fit solution.

Table 3

Orbital Elements for HD 95872b

\begin{tabular}{ll}
\hline \hline Parameter & Value \\
\hline Period (days) & $4375[169]$ \\
Mass $\left(M_{\text {jup }}\right)$ & $4.6[0.3]$ \\
Mean anomaly (deg) & $283[65]$ \\
Eccentricity & $0.06[0.04]$ \\
Longitude of pericenter $(\operatorname{deg} 0$ & $17[67]$ \\
Semiamplitude $\left(\mathrm{m} \mathrm{s}^{-1}\right)$ & $59[4]$ \\
Semimajor axis $(\mathrm{AU})$ & $5.2[0.1]$ \\
Periastron passage time $(\mathrm{JD})$ & $2,449,869[744]$ \\
Noise parameter, KECK data $\left(\mathrm{m} \mathrm{s}^{-1}\right)$ & $0.5[0.6]$ \\
Noise parameter, McDonald data $\left(\mathrm{m} \mathrm{s}^{-1}\right)$ & $8[2]$ \\
RV offset Keck/McDonald $\left(\mathrm{m} \mathrm{s}^{-1}\right)$ & $19[2]$ \\
\hline Stellar mass $\left(M_{\text {Sun }}\right)$ & 0.95 \\
rms (m s $\left.{ }^{-1}\right)$ & 7.90 \\
Jitter (best fit) $\left(\mathrm{m} \mathrm{s}^{-1}\right)$ & 4.80 \\
Epoch $(\mathrm{JD})$ & $2,453,073.87$ \\
Data points & 44 \\
Span of observations $(\mathrm{JD})$ & $2,453,073.87$ (2004 Apr) \\
& $2,457,153.62$ (2015 May)
\end{tabular}

Note. For parameters derived from the MCMC analysis, we report their median values and their mean absolute deviation (in brackets).

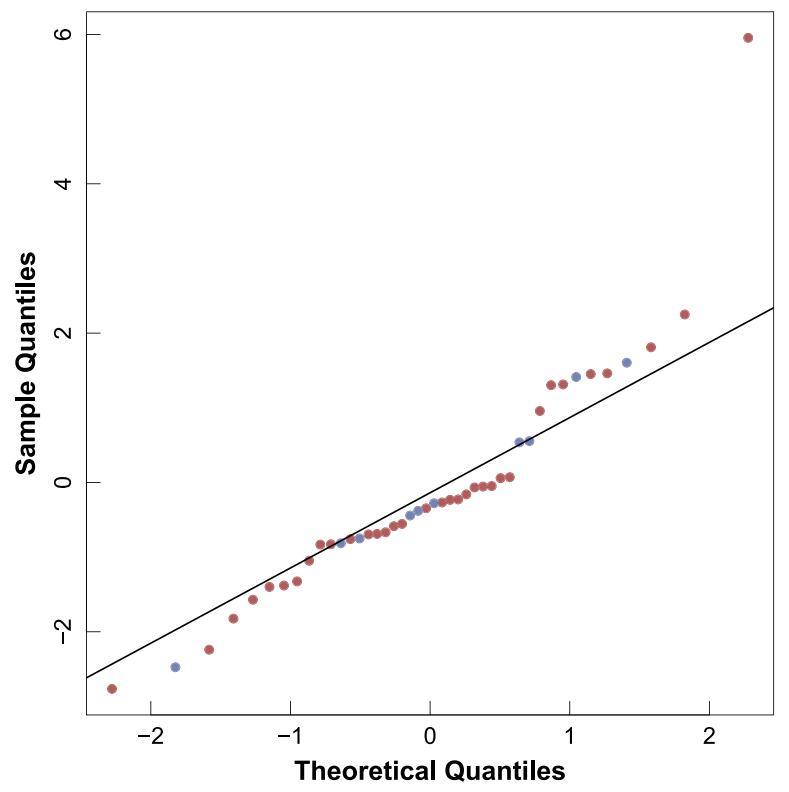

Figure 4. Quantile-quantile plot of the residuals from the one-planet model for HD 95872. Perfectly normally distributed residuals would fall on the solid line.

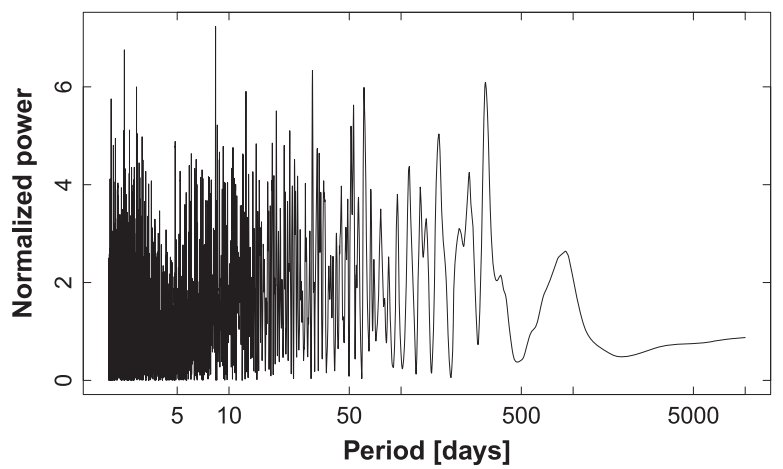

Figure 5. Lomb-Scargle periodogram of the residuals. All peaks have an FAP $>10 \%$.

\subsection{Stellar Activity Check}

The large amplitude of $\sim \pm 60 \mathrm{~m} \mathrm{~s}^{-1}$ of the detected RV signal makes it unlikely that a long-term magnetic cycle is responsible for it. The relative faintness of this $\operatorname{star}(V=9.9)$ leads to very low $\mathrm{S} / \mathrm{N}$ values in the blue spectral orders that contain the Ca II $\mathrm{H} \& \mathrm{~K}$ lines at $390 \mathrm{~nm}$. Therefore, we cannot determine a reliable time series of $S_{\mathrm{HK}}$ index measurements for this star using the Tull spectra. However, nine HIRES spectra have sufficient $\mathrm{S} / \mathrm{N}$ to obtain the $S_{\mathrm{HK}}$ index value. We calculate the $R_{\mathrm{HK}}^{\prime}$ value following Paulson et al. (2002). We find $R^{\prime}{ }_{\mathrm{HK}}=-5.46 \pm 0.044$ for HD 95872. This means that HD 95872 is an inactive star and that the planetary hypothesis for the detected RV variation is the preferred one. Figure 6 shows the $\mathrm{Ca}$ II $\mathrm{H} \& \mathrm{~K}$ lines from the HIRES spectrum with the highest $\mathrm{S} / \mathrm{N}$. There is nearly no chromospheric emission detectable in the line cores, in agreement with the very low value of $R^{\prime}{ }_{\text {HK }}$.

Stellar activity can also manifest itself as variation of the average line shape. We therefore measured the velocity span of the line bisector (BVS) in the iodine-free regions of our Tull spectra. We find a mean BVS value of $-0.05 \mathrm{~km} \mathrm{~s}^{-1}$ with an rms scatter of $0.077 \mathrm{~km} \mathrm{~s}^{-1}$. The average $1 \sigma$ error on the BVS results is $0.052 \mathrm{~km} \mathrm{~s}^{-1}$. We do not find any gross variability in 


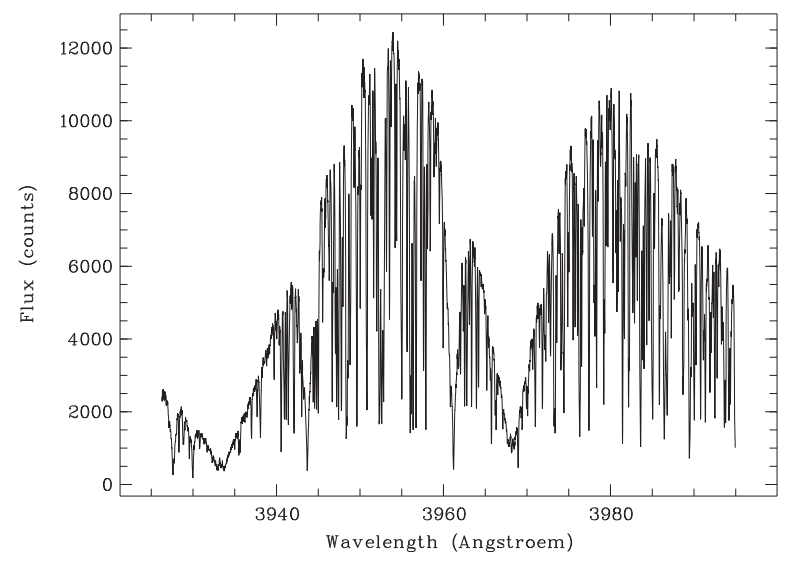

Figure 6. Ca II H \& $\mathrm{K}$ lines of HD 95872 in our best Keck/HIRES spectrum. The very low level of chromospheric emission in the line kernel shows that this star is quiet and inactive with $R_{\mathrm{HK}}^{\prime}=-5.46 \pm 0.044$.

the line bisectors that would cast doubt on the planetary origin of the signal. The average uncertainty of the BVS measurements is comparable to the detected RV signal, which limits the usefullness of this analysis. The large uncertainty of $\sim 50 \mathrm{~m} \mathrm{~s}^{-1}$ is - again - due to the low $\mathrm{S} / \mathrm{N}$ of spectra of this relatively faint target star.

\section{THE $\psi^{1}$ DRACONIS SYSTEM}

The $\psi^{1}$ Draconis system is a visual binary composed of an F5V primary $\left(\psi^{1}\right.$ Dra A, 31 Dra A, HR 6636, HD 162003, HIP 86614) and an F8V secondary star ( $\psi^{1}$ Dra B, 31 Dra B, HR 6637, HD 162004, HIP 86620) separated by about 30". 1 . At a distance of $22.2 \mathrm{pc}$, this corresponds to a sky-projected separation of approximately 667 AU. Previously, Toyota et al. (2009) reported evidence of an unseen companion orbiting the A component of the system, with a minimum mass of $50 M_{J}$. We have monitored both stars for long-term RV variability and also find evidence for a stellar-mass companion around the A component. Moreover, we discovered two planetary/substellar companions orbiting the B component. Thus, the $\psi^{1}$ Draconis system is at least a hierarchical triple system, with the primary having a low-mass, $\mathrm{K}$ - or M-dwarf companion and the secondary having two candidate planetary/substellar companions.

\section{1. $\psi^{1}$ Draconis $A$}

Table 4 presents the complete set of our RV observations for the primary star $\psi^{1}$ Dra A. The RV coverage spans nearly 15 $\mathrm{yr}$ of monitoring over $77 \mathrm{RV}$ measurements. The median internal uncertainty for our RV data is $\approx 15 \mathrm{~m} \mathrm{~s}^{-1}$, and the peak-to-peak velocity change is $>10,000 \mathrm{~m} \mathrm{~s}^{-1}$, typical for a stellar companion. The most recent RV measurements revealed that the star has passed the maximum of its RV orbit and is now approaching a periastron passage (see Figure 7).

We performed a similar orbit-fitting analysis to that in the case of HD 95872. The marginal distributions of the orbital elements are shown in Figure 8. The binary orbit due to the stellar companion to $\psi^{1}$ Dra A has a period of $P \approx 6600$ days, an eccentricity of $e \approx 0.67$, and a semiamplitude of $K \approx 5160 \mathrm{~m} \mathrm{~s}^{-1}$. These values are consistent with a low-mass stellar companion $\left(\psi^{1}\right.$ Dra $\left.\mathrm{C}\right)$ to the primary at an orbital separation of $a \approx 9$ AU. Table 5 summarizes the orbital elements that we determined from the RV data.
Table 4

Differential Radial Velocity Measurements for $\psi^{1}$ Draconis A (Sample)

\begin{tabular}{lccc}
\hline \hline Spectrum & BJD & $\begin{array}{c}\mathrm{dRV} \\
\left(\mathrm{m} \mathrm{s}^{-1}\right)\end{array}$ & $\begin{array}{c}\text { Uncertainty } \\
\left(\mathrm{m} \mathrm{s}^{-1}\right)\end{array}$ \\
\hline 1 & $2,451,809.6596$ & 1946.7 & 12.8 \\
2 & $2,451,809.6740$ & 1947.4 & 14.3 \\
3 & $2,452,142.6805$ & 1862.1 & 11.9 \\
4 & $2,453,319.6392$ & 2450.7 & 11.4 \\
5 & $\ldots$ & $\ldots$ & $\ldots$ \\
\hline
\end{tabular}

(This table is available in its entirety in machine-readable form.)
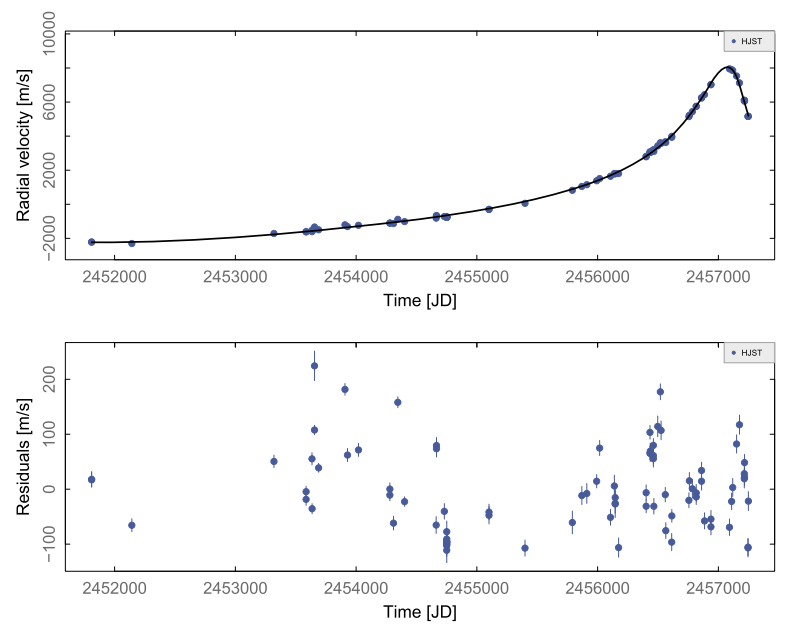

Figure 7. Top panel: our RV data for $\psi^{1}$ Dra A showing an eccentric binary orbit with a period of $P \approx 6650$ days (nearly $20 \mathrm{yr}$ ). Bottom panel: RV residuals from the binary orbit. We find a large excess scatter of $\approx 70 \mathrm{~m} \mathrm{~s}^{-1}$, indicating significant intrinsic stellar variability.

One striking feature of these orbital solutions are the large values of residual $\mathrm{RV}$ scatter around the fit considering our typical $\mathrm{RV}$ uncertainties of $\approx 15 \mathrm{~m} \mathrm{~s}^{-1}$. The models require an astrophysical noise term of $\approx 75 \mathrm{~m} \mathrm{~s}^{-1}$ to achieve a good fit. The Lomb-Scargle periodogram of the best-fit RV residuals (Figure 9) does not show any convincing periodic signals that could indicate additional companions in the system. However, with an F5V spectral type classification, $\psi^{1}$ Dra $\mathrm{A}$ is one of earliest spectral types in our target list. In the H-R diagram this star is located close to the red edge of the instability strip. We therefore examined the Hipparcos photometry (ESA 1997) of $\psi^{1}$ Dra A to search for stellar pulsations. The Fourier transform of the photometry is displayed in Figure 10. We find a peak at a period of $2.1 \mathrm{hr}\left(=11.29\right.$ cycles day $\left.^{-1}\right)$ with a modulation amplitude of $>4 \sigma$. This period value falls within the range of a few hours of typical $p$-mode oscillations of $\delta$ Scuti-type pulsators (e.g., Balona et al. 2015). We therefore suspect that these stellar oscillations are responsible for the large observed excess scatter.

In Section 5.3 we will discuss in more detail the detection of $\psi^{1}$ Dra $\mathrm{C}$, the stellar companion, by direct imaging. Owing to the small angular separation between $\psi^{1}$ Dra A and C, we expect that some of the residual scatter is also caused by contamination from light from the faint companion star that also entered through the spectrograph slit. In a companion paper (Gullikson et al. 2015) we successfully retrieve the Doppler signal of the low-level secondary spectrum and thus determine a double-lined spectroscopic orbital solution for $\psi^{1}$ Dra A/C. 

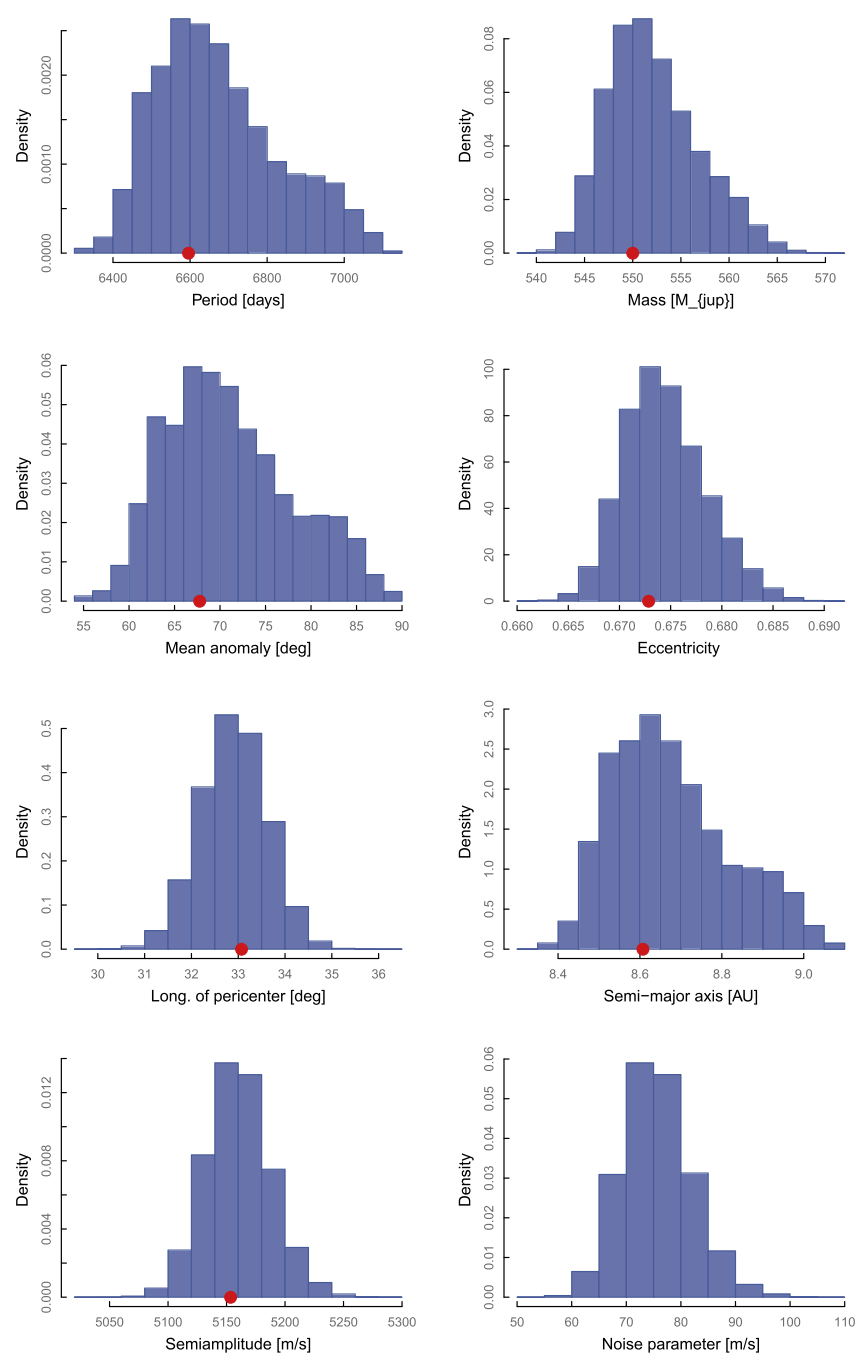

Figure 8. Marginal distributions of the orbital elements for the single-lined spectroscopic binary orbit of $\psi^{1}$ Draconis A, as computed by the MCMC algorithm. The red dot marks the value of the best-fit solution.

Table 5

Orbital Elements for the Single-lined Spectroscopic Binary Orbit of $\psi^{1}$ Draconis A and C

\begin{tabular}{|c|c|}
\hline Parameter & Value [Uncertainty] \\
\hline Period (days) & 6649 [160] \\
\hline Mass $\left(M_{\text {jup }}\right)$ & $551[5]$ \\
\hline Mean anomaly (deg) & $70[7]$ \\
\hline Eccentricity & $0.674[0.004]$ \\
\hline Long. of pericenter (deg) & $32.9[0.7]$ \\
\hline Semiamplitude $\left(\mathrm{m} \mathrm{s}^{-1}\right)$ & $5159[27]$ \\
\hline Semimajor axis (AU) & $8.7[0.1]$ \\
\hline Periastron passage time (JD) & $2450515[162]$ \\
\hline Noise parameter $\left(\mathrm{m} \mathrm{s}^{-1}\right)$ & $75[6]$ \\
\hline Stellar mass $\left(M_{\text {Sun }}\right)$ & 1.430 \\
\hline Chi-square & 85.673 \\
\hline Log likelihood & 486.353 \\
\hline $\mathrm{rms}\left(\mathrm{m} \mathrm{s}^{-1}\right)$ & 74.231 \\
\hline Jitter (best fit) $\left(\mathrm{m} \mathrm{s}^{-1}\right)$ & 72.617 \\
\hline Epoch (JD) & $2,451,809.660$ \\
\hline Data points & 85 \\
\hline \multirow[t]{2}{*}{ Span of observation (JD) } & $2,451,809.6596$ (2000 Sep) \\
\hline & $2,457,248.6070$ (2015 Aug) \\
\hline
\end{tabular}



Figure 9. Generalized Lomb-Scargle periodogram of the best-fit RV residuals from the binary orbital solution. The horizontal dashed lines show FAP levels of $10 \%, 1 \%$, and $0.1 \%$ respectively.

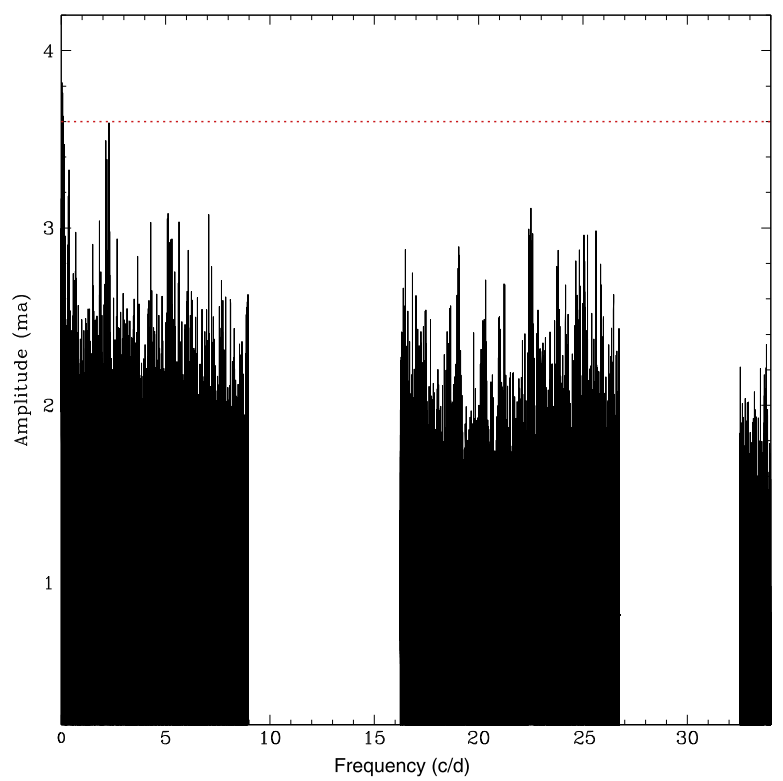

Figure 10. Fourier transform of the Hipparcos photometry of $\psi^{1}$ Dra A revealing a strong peak at a frequency of 11.29 cycles day $^{-1}(P=2.1 \mathrm{hr})$. The horizontal dashed line shows the $4 \sigma$ level $(\mathrm{FAP} \approx 0.001)$ of significance. The detected period of $2.1 \mathrm{hr}$ is typical for nonradial stellar oscillations of a classic $\delta$ Scuti variable.

Table 6

Differential Radial Velocity and Ca H \& K Observations for $\psi^{1}$ Dra B (Sample)

\begin{tabular}{lccccc}
\hline \hline Spectrum & BJD & $\begin{array}{c}\mathrm{dRV} \\
\left(\mathrm{m} \mathrm{s}^{-1}\right)\end{array}$ & $\begin{array}{c}\text { Uncertainty } \\
\left(\mathrm{m} \mathrm{s}^{-1}\right)\end{array}$ & $S_{\mathrm{HK}}$ & Uncertainty \\
\hline 1 & $2,451,066.7344$ & -48.8 & 6.1 & 0.155 & 0.0198 \\
2 & $2,451,121.6124$ & -48.5 & 3.8 & 0.161 & 0.0213 \\
3 & $2,451,271.9939$ & -39.1 & 7.3 & 0.163 & 0.0197 \\
4 & $2,451,329.8559$ & -33.4 & 5.5 & 0.162 & 0.0206 \\
5 & $2,451,360.8829$ & -39.1 & 4.2 & 0.162 & 0.0214 \\
6 & $2,451,417.7778$ & -24.5 & 5.0 & 0.173 & 0.0214 \\
7 & $2,451,451.6921$ & -24.9 & 6.1 & 0.167 & 0.0217 \\
8 & $\ldots$ & $\ldots$ & $\ldots$ & $\ldots$ & $\ldots$ \\
\hline
\end{tabular}

(This table is available in its entirety in machine-readable form.)

\section{2. $\psi^{1}$ Draconis $B$}

Table 6 presents the complete set of our RV observations for $\psi^{1}$ Dra B. The RV coverage spans approximately $16 \mathrm{yr}$ of monitoring over 135 measurements. The median internal uncertainty for our observations is $\approx 5.6 \mathrm{~m} \mathrm{~s}^{-1}$, and the peak- 
Table 7

Astrocentric Orbital Elements for $\psi^{1}$ Dra Bb

\begin{tabular}{|c|c|}
\hline Parameter & Value \\
\hline Period (days) & $3117[42]$ \\
\hline $\operatorname{Mass}\left(M_{\text {jup }}\right)$ & $1.53[0.10]$ \\
\hline Mean anomaly (deg) & 199 [7] \\
\hline Eccentricity & $0.40[0.05]$ \\
\hline Long. of pericenter (deg) & $64[9]$ \\
\hline Semiamplitude $\left(\mathrm{m} \mathrm{s}^{-1}\right)$ & $21[1]$ \\
\hline Semimajor axis (AU) & $4.43[0.04]$ \\
\hline Periastron passage time (JD) & $2449344[76]$ \\
\hline Noise parameter $\left(\mathrm{m} \mathrm{s}^{-1}\right)$ & $4.5[0.7]$ \\
\hline Quadratic trend $\left(\mathrm{m} \mathrm{s}^{-2}\right)$ & $-0.0000041[0.0000002]$ \\
\hline Linear trend $\left(\mathrm{m} \mathrm{s}^{-1}\right)$ & $0.032[0.002]$ \\
\hline Stellar mass $\left(\mathcal{M}_{\text {Sun }}\right)$ & 1.19 \\
\hline $\mathrm{rms}\left(\mathrm{m} \mathrm{s}^{-1}\right)$ & 7.048 \\
\hline Jitter (best fit) $\left(\mathrm{m} \mathrm{s}^{-1}\right)$ & 3.250 \\
\hline Epoch (JD) & $2,451,066.734$ \\
\hline Data points & 135 \\
\hline \multirow[t]{2}{*}{ Span of observations (JD) } & $2,451,066.7344$ (1998 Oct) \\
\hline & $2,457,248.6109$ (2015 Aug) \\
\hline
\end{tabular}

Note. For parameters derived from the MCMC analysis, we report their median values and their mean absolute deviation (in brackets).

to-peak velocity is $\approx 62 \mathrm{~m} \mathrm{~s}^{-1}$. The velocity scatter around the average $\mathrm{RV}$ is $\approx 14.3 \mathrm{~m} \mathrm{~s}^{-1}$.

\subsubsection{Companion Orbit Models}

The differential RV data for $\psi^{1}$ Dra $\mathrm{B}$ are plotted in Figure 11. The Lomb-Scargle periodogram (Figure 11) for the RV data shows two strong peaks at $P_{1} \approx 2381$ days and $P_{2}>6000$ days (longer than the time span of our observations). We model the second signal with two parameters representing a linear and a quadratic term (evaluated at the epoch of the fit).

Once the linear and quadratic trend terms are removed (Figure 12 ), a strong periodicity arises at $P \approx 3030$ days. The bootstrapped FAP probability is very low (FAP $<2 \times 10^{-5}$ ). We fit this periodicity with a model that simultaneously minimizes the linear and quadratic trend terms and the five orbital elements describing an eccentric orbit (period, mass, mean anomaly, eccentricity, and longitude of periastron). The best-fit model is shown in Figure 13. The data are well modeled by a Keplerian orbit of period $P=3117 \pm 42$ days and semiamplitude $K=20.6 \pm 1.4 \mathrm{~m} \mathrm{~s}^{-1}$. Together with the assumed stellar mass of $1.19 \mathcal{M}_{\odot}$, this implies a minimum mass of $\mathcal{M} \sin i=1.53 \pm 0.09 \mathcal{M}_{J}$ and a semimajor axis $a=4.43 \pm 0.04 \mathrm{AU}$. No compelling peaks are evident in the periodogram of the residuals (bottom panel in Figure 13). Figure 14 displays the RV data phased to the orbital period of the planet.

The data strongly favor a substantial eccentricity for $\psi^{1}$ Dra Bb $(e=0.40 \pm 0.05)$. The cross-validation algorithm (Andrae et al. 2010) corroborates the clear preference for an eccentric model $\left(\log \mathcal{L}_{\text {circular }} \approx 0.02\right.$ versus $\log \mathcal{L}_{\text {eccentric }} \approx-21.3$; lower is better).

The distribution of the orbital elements is shown in Figure 15. There is no strong correlation between any of the parameters of the fit, including between the trend parameters and the semiamplitude of the planet (bottom row). The derived stellar jitter is $4.5 \pm 0.7 \mathrm{~m} \mathrm{~s}^{-1}$. The distribution of the residuals shows


Figure 11. RV and Lomb-Scargle periodograms for $\psi^{1}$ Dra B. Top panel: our differential RV data. Middle panel: error-weighted Lomb-Scargle periodogram for $\psi^{1}$ Dra B. FAP levels are shown at the $10 \%, 1 \%$, and $0.1 \%$ level. Bottom panel: periodogram of the window function.

no evidence for unmodeled periodicities in the data. Indeed, we note that the normalized residuals are again very nearly normally distributed (Figure 16).

\subsubsection{Origin of the Trend}

In this section, we investigate the nature of the long-term trend observed in the data. In particular, we ascertain whether $\psi^{1}$ Dra A $\left(a \approx 600 \mathrm{AU}, \mathcal{M}_{A} \approx 1.38 \mathcal{M}_{\odot}, P \approx 9.4 \times 10^{4} \mathrm{yr}\right.$; Toyota et al. 2009) is the source of the long-term trend.

To model the long-term trend, we first assume that the gravitational pull is provided by an external perturber $\left(\psi^{1}\right.$ Dra $\mathrm{Bc}$ ) in a circular orbit. We fit the data by fixing the eccentricity of the perturber to zero and sampling periods between 4000 days and 15,000 yr. The top panel of Figure 17 shows the best fit for the mass of the perturber at each period sampled. The goodness of the fit (as measured by the rms of the residuals) is shown in the bottom panel. Beyond approximately $10^{4}$ days, the rms is flat and the period and mass of the perturber are degenerate. We note that component A cannot be 

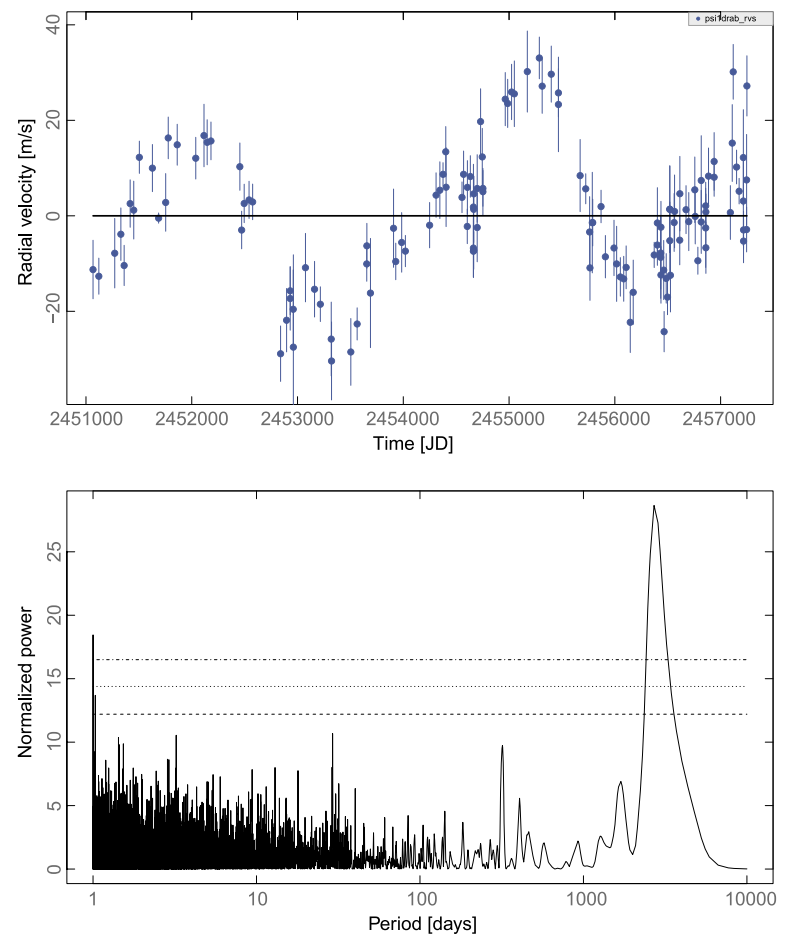

Figure 12. RV and Lomb-Scargle periodograms for $\psi^{1}$ Dra B, with the linear and quadratic trends removed. Top panel: relative RV data. Bottom panel: error-weighted Lomb-Scargle periodogram for $\psi^{1}$ Dra B. FAP levels are shown at the $10 \%, 1 \%$, and $0.1 \%$ level.

the source of the long-term trend, given the minimum mass required for $\mathrm{A}$ at the observed binary separation.

If we relax the assumption of a circular orbit for the external perturber, then the predicted mass of the perturber at each orbital period will be smaller at higher eccentricities (Figure 18). This is because at higher eccentricities and fixed periods, the curvature of the RV signal will be provided at the pericenter swing of the perturber. Therefore, the mass of the outer companion is determined by the pericenter distance $(q=a(1-e)$; Figure 18, bottom panel), as expected. Again, component $\mathrm{A}$ is not close or massive enough to produce the observed curvature.

\subsubsection{Stellar Activity Check}

We examined the Ca II $S_{\mathrm{HK}}$ values determined from the spectra of $\psi^{1}$ Dra B. The mean $S_{\mathrm{HK}}$ index for this star is $0.167 \pm 0.0008$, which is a typical value for a magnetically quiet star (e.g., the inactive star $\tau$ Ceti has $S_{\mathrm{HK}}=0.167 \pm 0.0013$ measured from our spectra). We find a linear correlation coefficient of 0.116 of the $S_{\mathrm{HK}}$ with the RV values. This translates to a probability of $p \approx 18 \%$ that the null hypothesis of no correlation is correct. Very small values of $p$ would indicate a correlation between the two quantities, but they are not strongly correlated.

Figure 19 shows the result of a period search in the $S_{\mathrm{HK}}$ data. No strong peaks and thus no significant periodicities are detected. We also note that there is virtually no power at the orbital period of planet $\mathrm{b}(P=3117 \pm 42$ days, indicated as a vertical dashed line in the figure). This indicates that the RV variations of $\psi^{1}$ Dra B are not caused by stellar activity. There is, however, some moderate power at periods exceeding our time baseline. This can be due to a low-level trend in magnetic
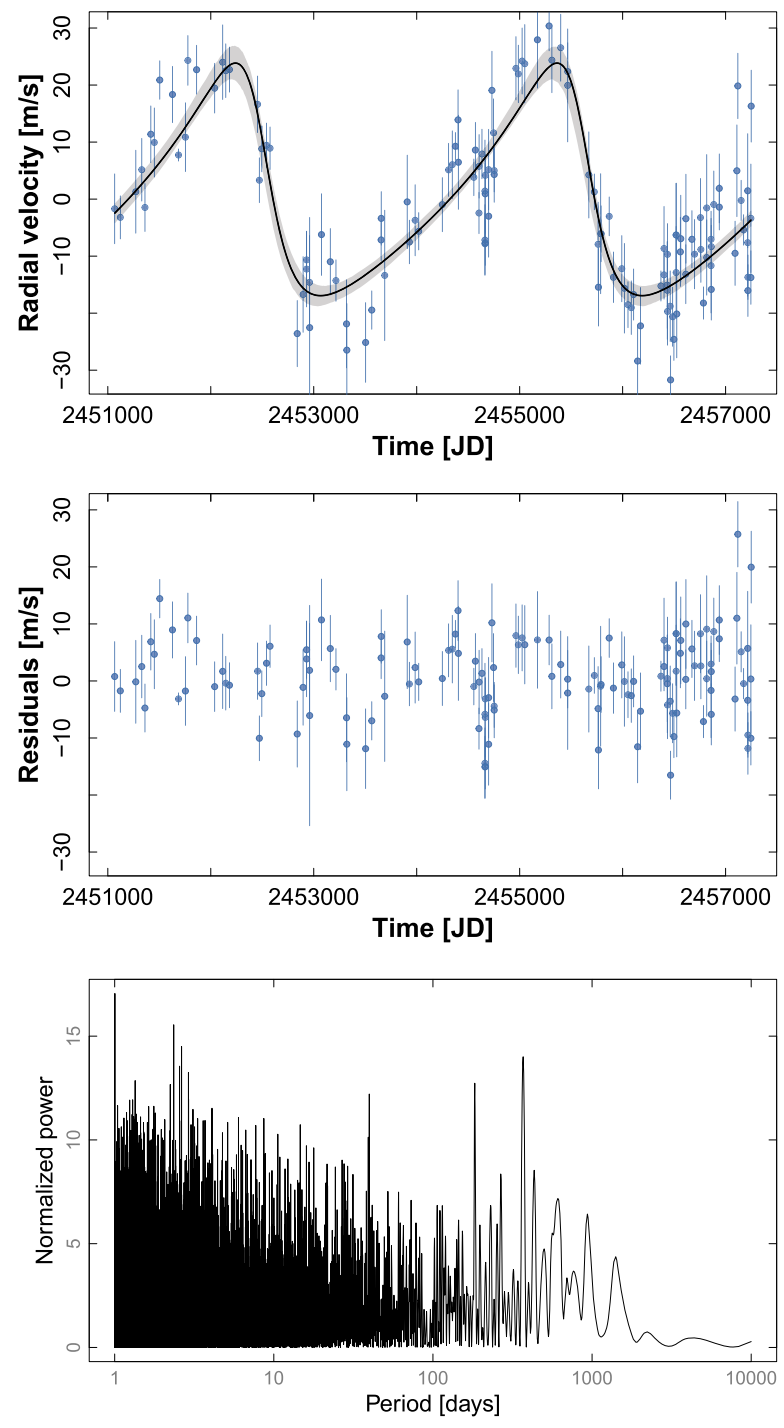

Figure 13. Best one-planet fit of the $\mathrm{RV}$ data set for $\psi^{1}$ Dra $\mathrm{B}$. Top: RV observations (linear and quadratic term subtracted) and one-planet best fit. The shaded area marks the 10\%-90\% percentiles of the RV curves sampled from the MCMC trials and indicates the range of the models compatible with the data. Middle: residuals from the one-planet best fit. Bottom: periodogram of the residuals from the one-planet best fit.

activity of the star, possibly caused by a very long activity cycle.

\subsubsection{Dynamical Stability Analysis}

A number of recent studies have highlighted the value of examining the dynamical behavior of candidate planetary systems as a critical part of the planet discovery process (e.g., Horner et al. 2012a, 2012b; Robertson et al. 2012a, 2012b; Wittenmyer et al. 2012b, 2014a). We therefore chose to carry out a detailed dynamical study of the stability of the proposed $\psi^{1}$ Dra B system, as a function of the orbit of the relatively unconstrained outer body.

As in our earlier work, we carried out a total of 126,075 individual simulations of the $\psi^{1}$ Dra B planetary system, following the evolution of the two candidate planets for a period of $100 \mathrm{Myr}$ using the Hybrid integration package within the $n$-body dynamics program MERCURY (Chambers 1999). For these simulations, we have ignored the binary companion 

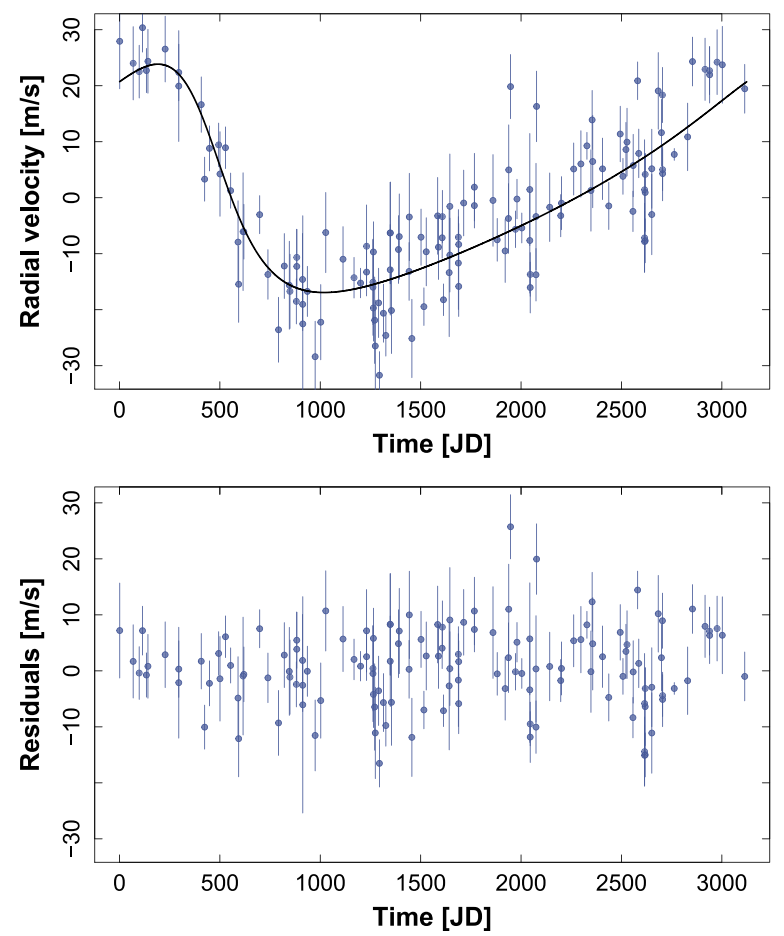

Figure 14. Phased best one-planet fit of the RV data set for $\psi^{1}$ Dra B. Top: RV observations (linear and quadratic term subtracted) and one-planet best fit. Bottom: residuals from the one-planet best fit.

$\psi^{1}$ Dra A-with a projected orbital separation of $\sim 600 \mathrm{AU}$, it is expected to have a negligible effect on the dynamics of the two planets considered here. In the case that one of the planets collided with the other, or was either flung into the central body or ejected from the system, the time at which that event occurred within the simulation was recorded, and the simulation was then terminated. This allowed us to create a map of the dynamical stability of the $\psi^{1}$ Dra B system as a function of the initial semimajor axis and eccentricity of the outermost planet, as can be seen in Figure 20.

In each of our 126,075 simulations, we used the same initial conditions for the orbit of the innermost planet, as given in Table 6. For $\psi^{1}$ Dra B c, we systematically varied the semimajor axis, eccentricity, argument of periastron $(\omega)$, and mean anomaly $(M)$ to create a grid of $41 \times 41 \times 15 \times 5$ possible orbital solutions for that planet. In the case of the planet's semimajor axis, $\omega$, and mean anomaly, we sampled the full $\pm 3 \sigma$ range around the nominal best-fit values for each parameter. The parameters we used for planet $\mathrm{c}$ were as follows: $a=16.2 \pm 3.7 \mathrm{AU}, \omega=299^{\circ} \pm 10^{\circ}$, and mean anomaly $M=299^{\circ} \pm 10^{\circ}$. For the eccentricity, we sampled 41 equally spaced values ranging between 0.0 and 0.5 . This allowed us to investigate in some depth the influence that the eccentricity of the planet's orbit will have on the system's stability.

The results of our simulations can be seen in Figure 20. At each of the $a-e$ locations in that figure, the lifetime given is the mean of 75 individual runs, sampling the full $\omega-M$ parameter space. Most readily apparent in Figure 20 is that the nominal best-fit orbit is located in a broad region of orbital stability. Indeed, all solutions within $\pm 1 \sigma$ of the best-fit semimajor axis are dynamically stable, unless the initial orbital eccentricity is in excess of 0.2. This is not surprising: the relatively sharp delineation between stable and unstable orbits that can be seen
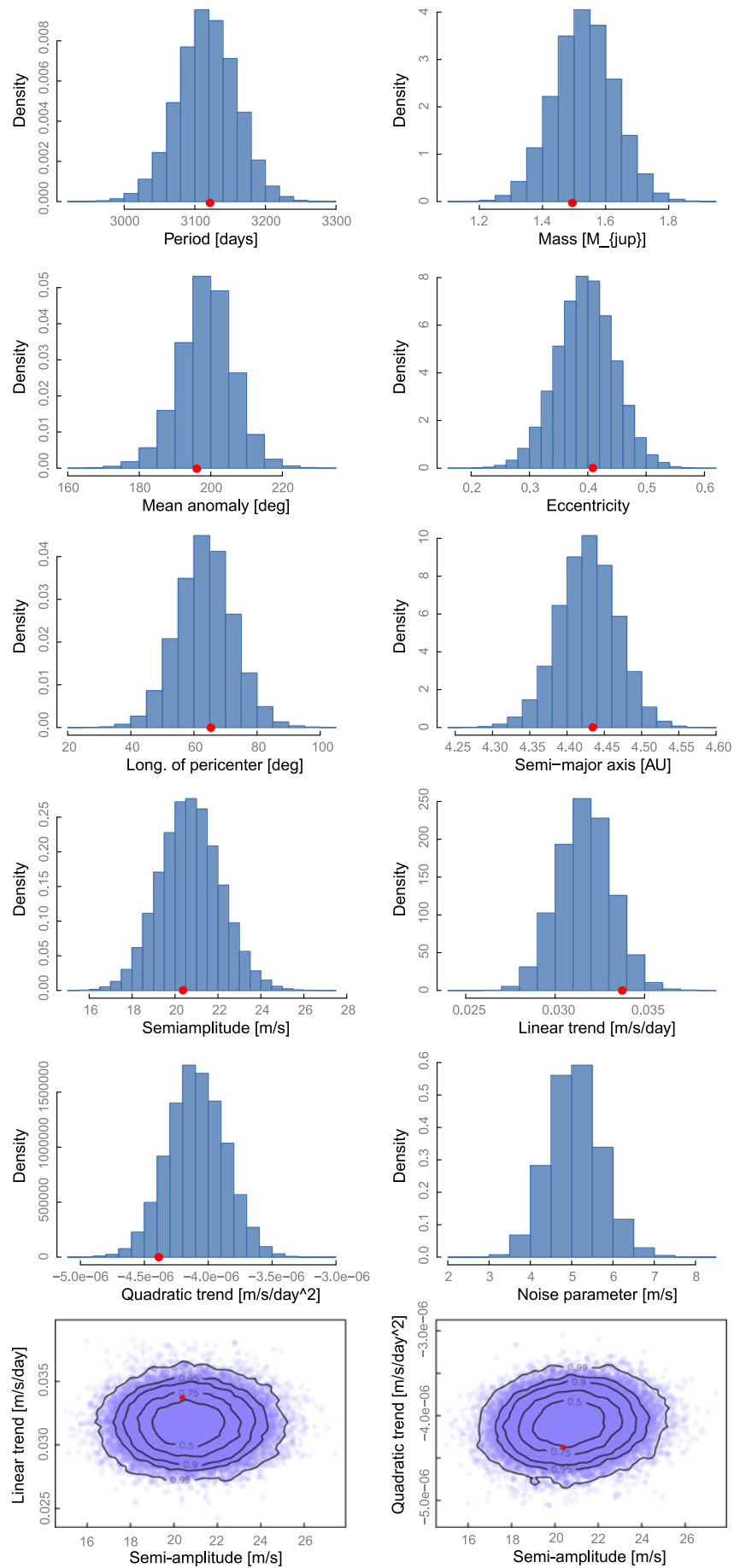

Figure 15. Marginal distributions of the orbital elements for the one-planet model, as computed by the MCMC algorithm. The red dot marks the value of the best-fit solution. The bottom row shows a contour plot of the planet semiamplitude $K$ vs. the linear and quadratic trend parameters.

curving upward from an origin at ( $a \sim 9, e \sim 0$ ) is a line of almost constant periastron distance and separates those orbits on which the planets cannot experience close encounters from those on which they can (and do). Following Chambers et al. (1996), we can determine the mutual Hill radius of the two companions at various semimajor axes (using their Equation (1)). Doing this, we note that when $\psi^{1} \operatorname{DraB} \mathrm{c}$ is located at $a=9 \mathrm{AU}$, the mutual Hill radius of the two companions is $\sim 1.02 \mathrm{AU}$, meaning that their orbits would be separated by less 


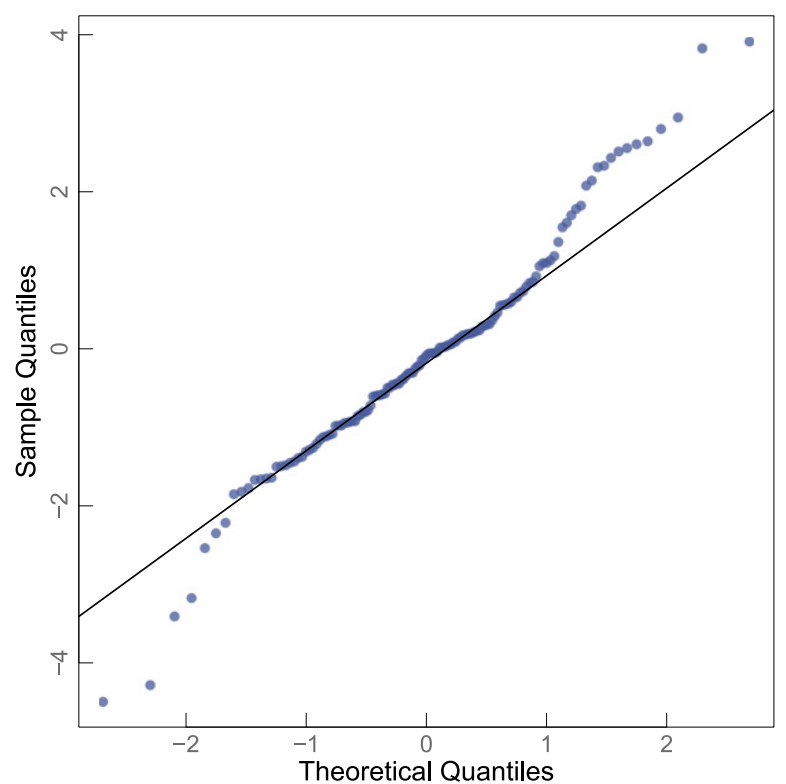

Figure 16. Quantile-quantile plot of the residuals from the one-planet model. Perfectly normally distributed residuals would fall on the solid line.


Figure 17. Top: correlation between the period and the mass of an outer body in a circular orbit that best fits the trend in the RV data. The red points correspond to systems that were unstable over a $10^{6}$ yr period. The black diamond marks the semimajor axis and mass of component $\psi^{1}$ Dra A from Toyota et al. (2009). Bottom: rms of the residuals for the best fit at each orbital period of the outer companion. At periods larger than $\approx 10^{4}$ days, the marginal distributions of the period and mass of the outer companion are flat.

than 5 mutual Hill radii. More critically, however, this situation would allow the two companions to approach one another within two mutual Hill radii should a close encounter happen while $\psi^{1}$ Dra B b (with its moderately large orbital eccentricity of 0.42 ) were close to apastron.

A few other noteworthy features can be readily observed in Figure 20. Interior to the broad area of stability lies a narrow
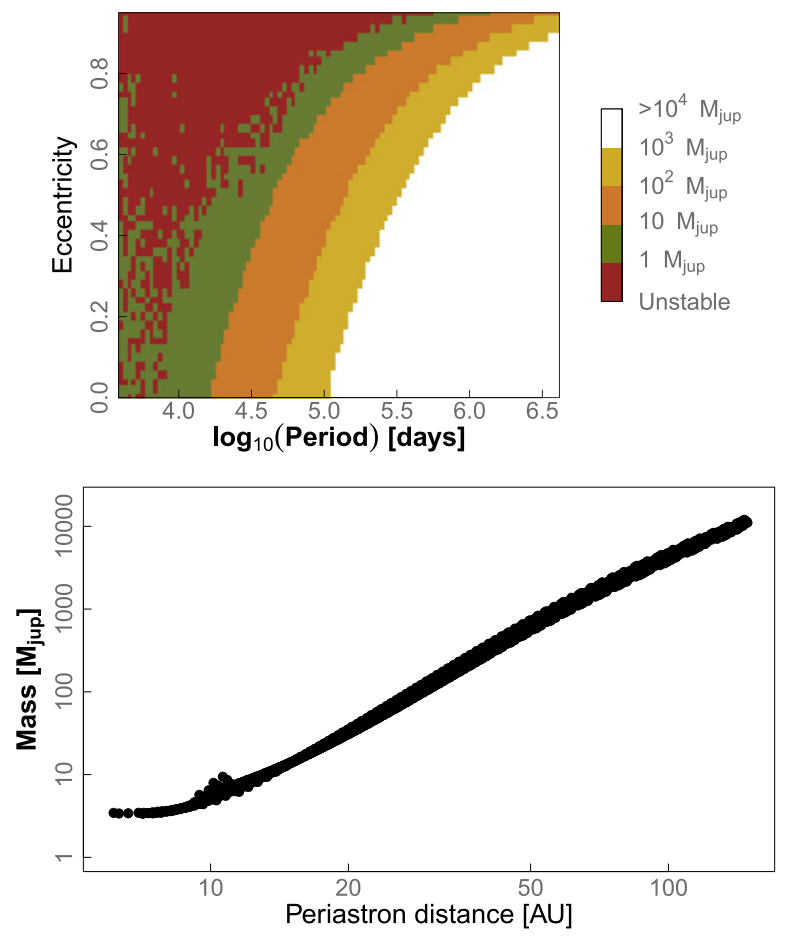

Figure 18. Top: contours of best-fit masses for the outer perturber, computed over a grid of fixed periods and eccentricities. Systems unstable within $10^{5}$ $\mathrm{yr}$ are marked in red. Bottom: relationship between the periastron distance and the mass of the outer perturber.

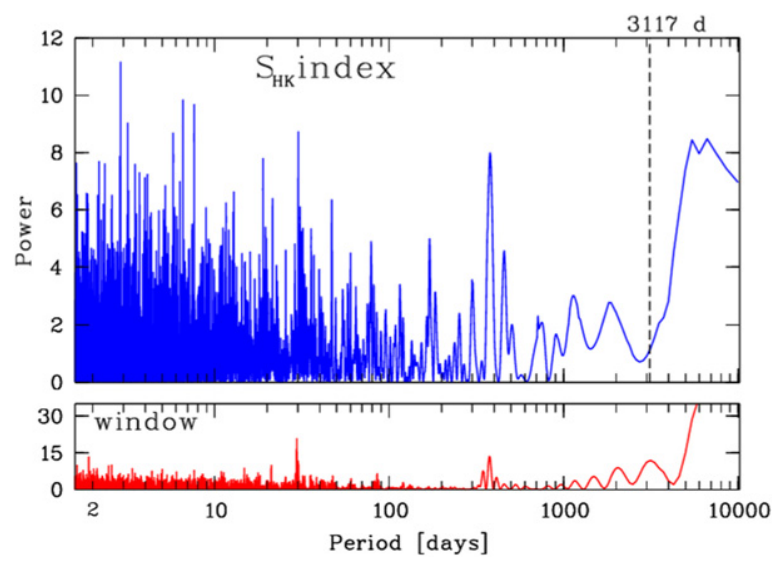

Figure 19. Lomb-Scargle periodogram of the Ca II $S_{\mathrm{HK}}$ index values of $\psi^{1}$ Dra $\mathrm{B}$ (top) and the window function of our observations. The vertical dashed line indicates the orbital period of planet b. No power is detected at the planet's period. There is moderate power at periods exceeding our time baseline ( $>6000$ days). This could be due to a low-level long-term trend in the magnetic activity of this star.

island of stability at $a \sim 7$ AU. Orbits in this region can be protected from destabilization by the influence of the mutual 2:1 mean-motion resonance between the two companions. Given an initial semimajor axis for $\psi^{1}$ Dra B b of $4.31 \mathrm{AU}$, a perfect 2:1 commensurability between the orbits of the two planets would occur at $a_{c} \sim 6.84 \mathrm{AU}$, so long as the initial architecture of the system is appropriate and the eccentricity of the orbit of $\psi^{1}$ Dra B c is not too large. Such islands of resonant stability are not uncommon and are thought to ensure the stability of several known exoplanetary systems (e.g., Wittenmyer et al. 2012a, 2014b). 


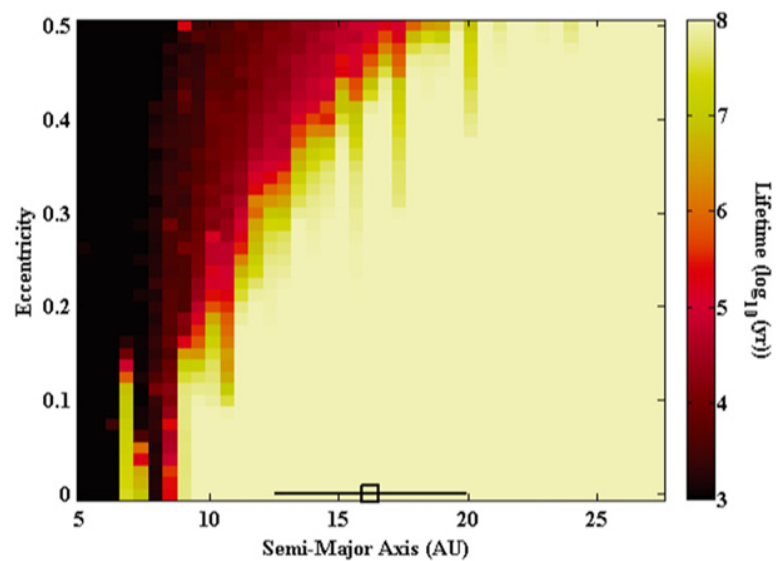

Figure 20. Dynamical stability map in semimajor axis and eccentricity space for the outer companion in the $\psi^{1}$ Dra B system. Dark areas represent unstable regions (see text for details).

Finally, a number of "bites" can be seen taken out of the broad region of stability-vertical strips of lower-than-average stability dotted at regular intervals through the whole range of semimajor axes examined (with the most prominent visible at $a \sim 11 \mathrm{AU})$. These represent locations where resonant interactions between the two companions act to destabilize, rather than stabilize, their orbits. These features serve as a reminder that even when two planets are well separated in their orbits around a given star, their orbits should still be checked for dynamical verisimilitude.

\subsection{Direct Imaging}

We observed both the A and B components of the $\psi^{1}$ Draconis system separately with the Differential Speckle Survey Instrument (DSSI) at the Gemini North telescope on 2014 July 19 UT. DSSI is a two-channel speckle camera described in Horch et al. (2009), which yields diffractionlimited information in two passbands simultaneously. A 1000frame sequence was recorded by each channel on each component. All frames were $60 \mathrm{~ms}$ exposures and had a format of $256 \times 256$ pixels. The seeing for both observations was 0 !" 65 . The image scale is approximately 0 ". 011 per pixel for both cameras.

We reduced and analyzed the results as follows. We form the average autocorrelation and average triple correlation of the set of speckle frames, and from these we estimate both the magnitude and phase of the Fourier transform of the source. The former must be deconvolved by a point-source observation in general; in the case of the data here, we constructed a point source matching the elevation and azimuth of the source by taking an observation of a point source at very high elevation and correcting it for the atmospheric dispersion expected for the elevation and azimuth of the science target. After the deconvolution, the magnitude and phase are assembled in the Fourier plane, low-pass filtered to suppress high-frequency noise, and inverse-transformed to arrive at a reconstructed (i.e., diffraction-limited) image of the target. More information about the reduction method with the current EMCCD cameras used with DSSI can be found in Horch et al. (2011).

With the reconstructed image in hand, we attempt to find companions by first examining the image. The images are shown in Figure 21. This yielded a strong stellar candidate at
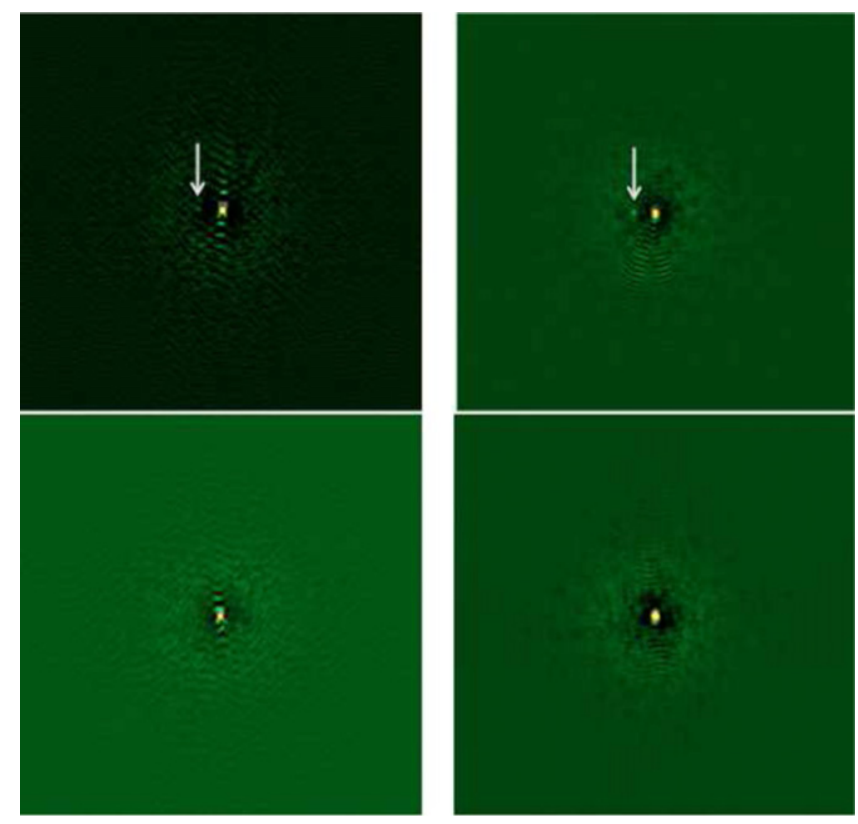

Figure 21. Reconstructed images of $\psi^{1}$ Draconis A and B taken with the Differential Speckle Survey Instrument on the Gemini North telescope, 2014 July 19 UT. Each frame is 2 ". $8 \times 2$ ". 8 in size. Top left: $\psi^{1}$ Draconis A at $692 \mathrm{~nm}$. Top right: $\psi^{1}$ Draconis A at $880 \mathrm{~nm}$. Bottom left: $\psi^{1}$ Draconis B at $692 \mathrm{~nm}$. Bottom right: $\psi^{1}$ Draconis B at $880 \mathrm{~nm}$. A faint very red companion is visible to the left of the primary star in the A images; the separation is 0 ." 16 .

approximate separation of 0 ". 16 from the primary star for $\psi^{1}$ Dra A, but no candidates for $\psi^{1}$ Dra B.

We then also computed a detection limit curve for the image; that is, a curve showing the largest magnitude difference that could be detected as a function of separation from the central star in the image. To construct the curve, we choose a set of concentric annuli centered on the central star and determine the statistics of the local maxima (peaks) occurring inside the annulus. If a peak in the annulus has a value of more than five times the sigma of all of the peaks above the average value of the peaks, we consider it to be a definitive detection of a stellar companion. Details of this process for Gemini data can be found in, e.g., Horch et al. (2012).

Figure 22 shows the detection limit curves for the four reconstructed images. The line in these figures is the $5 \sigma$ detection limit. If a source is a formally above a $5 \sigma$ detection, it would appear as a square data point lying below the curve. For $\psi^{1}$ Dra B, there are no plausible sources. For $\psi^{1}$ Dra A, we see a nearly $5 \sigma$ detection of a second star at a separation of about 0 .' 16 from the primary in the $880 \mathrm{~nm}$ image. In the $692 \mathrm{~nm}$ image, the same peak appears, but it is not as close to the $5 \sigma$ line. Looking again at the two images, we see that these data correspond in both cases to the peak at pixel $(114,129)$. Since both images show the same peak in the same spot, we are very confident that this component is stellar in nature. In these images north is essentially up and east is to the left, although there is a tilt of the celestial coordinates relative to pixel axes of about 5 degrees.

We next used our power spectrum fitting routine to determine the separation, position angle, and magnitude difference of the secondary. The results are summarized in Table 8 , when deconvolving by the calculated point sources described above. 

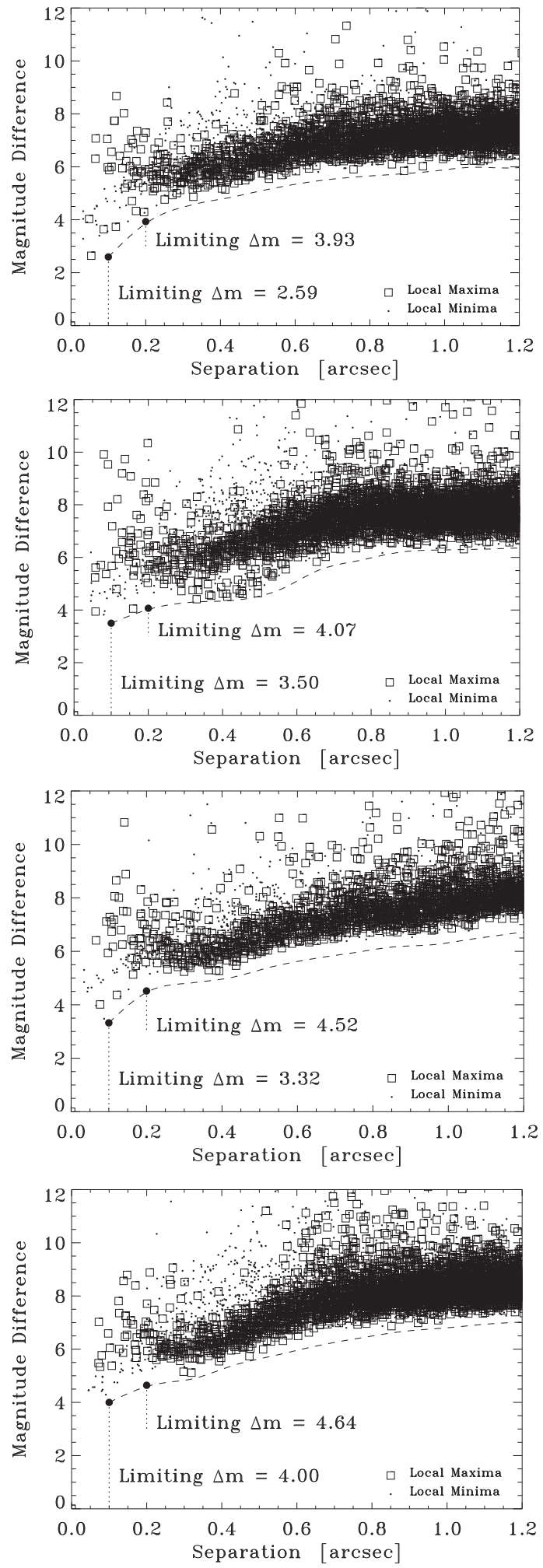

Figure 22. Detection limit curves as a function of separation for (first panel) $\psi^{1}$ Dra A at $692 \mathrm{~nm}$, (second panel) $\psi^{1}$ Dra A at $880 \mathrm{~nm}$, (third panel) $\psi^{1}$ Dra B at $692 \mathrm{~nm}$, and (fourth panel) $\psi^{1}$ Dra B at $880 \mathrm{~nm}$. These data are derived from the speckle image reconstructions as described in the text. In all plots, the squares are the implied magnitude differences from the central star for all local maxima in the reconstructed image, and solid points are the same drawn for the absolute values of all local minima (plotted to compare with the distribution of maxima). The dashed line indicates the magnitude difference for a peak that would be $5 \sigma$ above the average local maximum value, as judged from the statistics of the distribution itself. A detection of a companion at formal significance greater than $5 \sigma$ will lie below the line.
Table 8

Results of Imaging for $\psi^{1}$ Dra A, Using Generic Point-source Deconvolution

\begin{tabular}{cccc}
\hline \hline $\begin{array}{c}\text { Filter } \\
(\mathrm{nm})\end{array}$ & $\begin{array}{c}\text { Position Angle } \\
(\mathrm{deg})\end{array}$ & $\begin{array}{c}\text { Separation } \\
(\operatorname{arcsec})\end{array}$ & $\begin{array}{c}\text { Magnitude Difference } \\
(\mathrm{mag})\end{array}$ \\
\hline 692 & 91.8 & 0.155 & 4.13 \\
880 & 91.5 & 0.158 & 3.80 \\
\hline
\end{tabular}

Table 9

Results of Imaging for $\psi^{1}$ Dra A, Using $\psi^{1}$ Dra B Point-source Deconvolution

\begin{tabular}{cccc}
\hline \hline $\begin{array}{c}\text { Filter } \\
(\mathrm{nm})\end{array}$ & $\begin{array}{c}\text { Position Angle } \\
(\mathrm{deg})\end{array}$ & $\begin{array}{c}\text { Separation } \\
(\operatorname{arcsec})\end{array}$ & $\begin{array}{c}\text { Magnitude Difference } \\
(\mathrm{mag})\end{array}$ \\
\hline 692 & 91.8 & 0.156 & 4.22 \\
880 & 91.3 & 0.158 & 3.71 \\
\hline
\end{tabular}

Since $\psi^{1}$ Dra B is not resolved in our images, we also used it as the point source to deconvolve the images of $\psi^{1}$ Dra A, and in doing the power spectrum fitting that way, we obtain the results summarized in Table 9.

The differences between these numbers and the above give an estimate for the internal precision of the measurement technique. In looking at the power spectra for each file, we also see clear fringes that match the location shown in the reconstructed image. This gives an additional layer of confidence that we have detected a real stellar companion.

\subsection{Comparison of Elemental Abundances}

\subsubsection{Planet Signatures in Stellar Abundances}

An independent stellar parameter and detailed (multielement) chemical composition analysis for both stars in the $\psi^{1}$ Draconis system was carried out in order to search for chemical abundance anomalies that could be related to planet formation processes, as suggested by a number of recent studies. In their highly precise spectroscopic studies of solar twin stars, Meléndez et al. (2009) and Ramírez et al. (2009) have found the Sun to be slightly deficient in refractory elements, attributing this observation to the formation of rocky bodies in the solar system. They suggest that these objects captured the refractory elements that would have otherwise ended up in the Sun. In related work, Ramírez et al. (2011) and Tucci Maia et al. (2014) have found that the two solar-analog components of the 16Cygni binary system have slightly different overall metallicities and have attributed this effect to the formation of the gas giant planet that orbits 16 Cygni B (Cochran et al. 1997).

The rocky planet formation hypothesis for the refractory element depletion seen in the Sun has been challenged by González Hernández et al. (2010, 2013), while Schuler et al. (2011) have found no chemical abundance differences for the 16 Cygni stars. Thus, further investigation of other relevant stellar systems could shed light on this problem. The $\psi^{1}$ Draconis system is an interesting target in this context. Although not similar to the Sun, these stars are similar to each other, which is favorable to high-precision relative chemical composition analysis. Our $\psi^{1}$ Dra A spectrum is contaminated by the light from the stellar companion at the $1 \%$ level. 


\subsubsection{Atmospheric Parameter Determination}

We acquired very high $\mathrm{S} / \mathrm{N}$ spectra of the $\psi^{1}$ Draconis stars with the Tull spectrograph on the $2.7 \mathrm{~m}$ Telescope at McDonald Observatory on 2014 April 21. At $6000 \AA$, these spectra have $\mathrm{S} / \mathrm{N} \simeq 500$ per pixel and a spectral resolution $R=60,000$. These spectra are not part of the RV planet search data set; they were acquired specifically for the purpose of carrying out a detailed, strict differential atmospheric parameter and chemical abundance analysis. As described below, we analyzed $\psi^{1}$ Dra A relative to $\psi^{1}$ Dra B, but we also tested our differential calculations using a solar spectrum as reference. The latter was taken from a previous observing run (2013 December 18) in which reflected sunlight from the asteroid Vesta was used to collect a high-S/N (S/N $\simeq 350$ at $6000 \AA)$ solar spectrum with the same instrument/telescope and identical configuration.

Equivalent widths of $73 \mathrm{Fe}_{\mathrm{I}}$ lines and $18 \mathrm{Fe}$ II lines were measured by fitting Gaussian profiles to the observed spectral lines in the $\psi^{1}$ Draconis stars' and solar spectra using the splot task in IRAF. The line list and atomic parameters adopted are those by Ramírez et al. (2013). The uncertainty of the adopted $\log g f$ values and whether those were taken from laboratory measurements or calibrated using reference spectra (i.e., "astrophysical" values) are irrelevant in the strict differential approach implemented here. As mentioned above, the $\psi^{1}$ Dra A spectrum is contaminated at the $1 \%$ level by its stellar companion. We noticed this minor contamination in our spectra and attempted to remove it in our equivalent width measurements by using the "deblend" feature of splot whenever possible or by excluding sections of line wings in the line profile fits. Nevertheless, we expect the equivalent widths measured for $\psi^{1}$ Dra A to be less precise than those of $\psi^{1}$ Dra B, not only because of the spectral contamination, but also because of its somewhat faster projected rotational velocity.

The equivalent widths of each of the $\psi^{1}$ Draconis stars and the Sun were employed to calculate iron abundances using the abfind driver of the MOOG spectrum synthesis code, adopting Kurucz's odfnew grid of model atmospheres interpolated linearly to the assumed atmospheric parameters of each star. Then, on a line-by-line basis, differential iron abundances relative to the Sun were computed for the $\psi^{1}$ Draconis stars. The stellar parameters of the $\psi^{1}$ Draconis stars were modified iteratively until correlations of the iron abundance with excitation potential and reduced equivalent width disappeared and until the mean abundance of iron derived from Fe I and Fe II lines separately agreed. This procedure is standard in stellar spectroscopy (see Section 3.3), and it is sometimes referred to as the excitation/ionization equilibrium method of stellar parameter determination. To be more specific, hereafter we refer to this technique as the "iron-line-only" method. The particular implementation used here, including the error analysis, is described in detail in Ramírez et al. (2014; Section 3.1 and references therein).

The atmospheric parameters of the $\psi^{1}$ Draconis stars, derived as described above, are given in the first two rows of Table 10. The errors listed in that table are formal, i.e., they represent the precision with which we are able to find a self-consistent solution for the parameters, but do not take into account possible systematic errors. The $\psi^{1}$ Draconis stars are both significantly warmer than the Sun. Thus, we expect the analysis using the solar spectrum as reference to be affected by systematic errors in a nonnegligible way. Since we are
Table 10

Atmospheric Parameters of the $\psi^{1}$ Draconis Stars

\begin{tabular}{ccccc}
\hline \hline Star & $T_{\text {eff }}$ & $\log g$ & {$[\mathrm{Fe} / \mathrm{H}]^{\mathrm{a}}$} & References \\
\hline A & $6546 \pm 56$ & $3.90 \pm 0.14$ & $-0.10 \pm 0.04( \pm 0.07)$ & Sun \\
B & $6213 \pm 20$ & $4.35 \pm 0.05$ & $+0.00 \pm 0.01( \pm 0.04)$ & Sun \\
\hline A & $6544 \pm 42$ & $3.90 \pm 0.11$ & $-0.10 \pm 0.03( \pm 0.05)$ & B \\
\hline
\end{tabular}

Note.

${ }^{\mathrm{a}}$ The error bars in parentheses correspond to the $1 \sigma$ line-to-line scatter.

interested in the relative elemental abundances of the two $\psi^{1}$ Draconis stars, we could attempt to reduce these formal errors and also minimize the potential systematics, by analyzing $\psi^{1}$ Dra A using $\psi^{1}$ Dra B as the reference star. Adopting the parameters derived for $\psi^{1}$ Dra B using the Sun as reference (row 2 in Table 10), we computed the parameters of $\psi^{1}$ Dra A given in row 3 of Table 10. Note that the formal errors reduced, but the average values of the parameters were not significantly affected. This demonstrates that, when using the Sun as reference, systematic errors are introducing line-to-line scatter to the iron abundances of the $\psi^{1}$ Draconis stars.

In the last step we implicitly assumed that the parameters of $\psi^{1}$ Dra B derived using the Sun as reference are reliable. We tested this assumption by computing those parameters using independent techniques. For $T_{\text {eff }}$, we employed the effective temperature-color calibrations by Casagrande et al. (2010). For $\log g$, we used the stars' trigonometric parallaxes as given in the Hipparcos catalog, along with the Yonsei-Yale theoretical isochrone grid. Details on these techniques and the implementation used here are also provided in Ramírez et al. (2014; Sections 5.1 and 5.3 and references therein).

Using the $[\mathrm{Fe} / \mathrm{H}]$ values from Table 10 , the Casagrande et al. (2010) $T_{\text {eff }}$ calibrations for the $(B-V),(b-y)$, and $\left(B_{\mathrm{T}}-V_{\mathrm{T}}\right)$ colors provide mean values of $6519 \pm 20 \mathrm{~K}$ for $\psi^{1}$ Dra A and $6194 \pm 21 \mathrm{~K}$ for $\psi^{1}$ Dra B. Both these values are in agreement within formal error with those computed from the iron lines only (i.e., with the parameters given in Table 10). Moreover, their difference is $325 \mathrm{~K}$ according to the $T_{\text {eff }}$-color calibrations and $333 \mathrm{~K}$ according to the iron line analysis. This test confirms that the $T_{\text {eff }}$ adopted for $\psi^{1}$ Dra B in the strict differential analysis for $\psi^{1}$ Dra $\mathrm{A}$ is reliable.

The trigonometric $\log g$ values were computed using the $T_{\text {eff }}$ from the color calibrations, thus making them completely independent of the iron-line-only analysis. We calculated $\log g=4.02 \pm 0.02$ for $\psi^{1}$ Dra A and $\log g=4.32 \pm 0.02$ for $\psi^{1}$ Dra B. The spectroscopic (iron-line-only) $\log g$ of $\psi^{1}$ Dra A appears slightly low, yet it is still in marginal agreement with the trigonometric value within formal error. However, for $\psi^{1}$ Dra $\mathrm{B}$, the agreement is excellent, which also suggests that the $\log g$ adopted for $\psi^{1}$ Dra B in the strict differential analysis of $\psi^{1}$ Dra $\mathrm{A}$ is reliable.

As part of this calculation, we also computed age probability distributions for these stars using the same Yonsei-Yale isochrone set. We found most probable ages of 2.3 and 2.5 Gyr for psi1 Dra A and psi1 Dra B, respectively, both with precision errors of order $0.3 \mathrm{Gyr}$. The details of this calculation are explained in Ramírez et al. (2014, their Section 4.5). Note in particular that the errors do not include systematic uncertainties from the stellar models. The ages quoted above are somewhat younger than that given in Table 1 for psi1 Dra B (3.3 Gyr), but note that the latter has a much larger error bar 


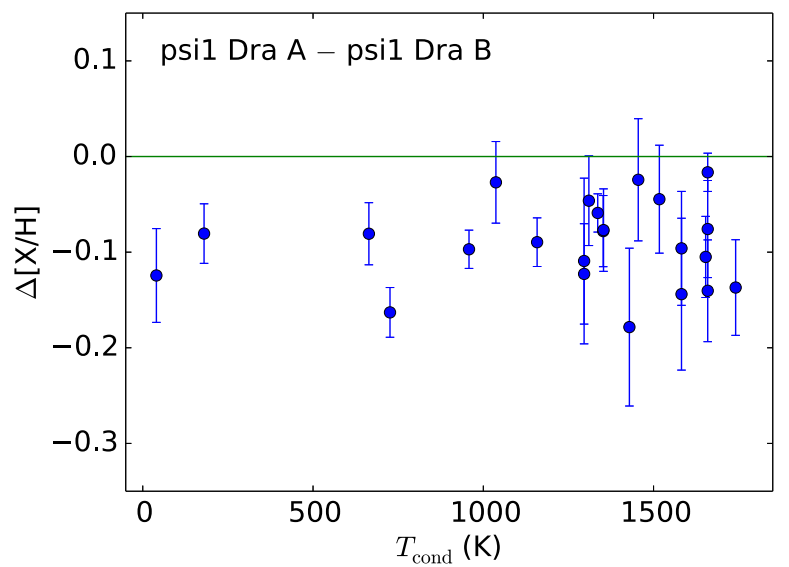

Figure 23. Elemental abundance difference between $\psi^{1}$ Dra $\mathrm{A}$ and $\psi^{1}$ Dra B as a function of the elements' condensation temperatures.

owing to the less precise stellar parameters employed. In fact, this age is consistent within error with the values listed here. In any case, the ages determined with the parameters measured as described in this section, which are based on higher-quality spectra, lead to consistent ages for psi1 Dra A and psi1 Dra B, as expected for a binary system.

\subsubsection{Multi-element Abundance Analysis}

Equivalent widths of spectral lines due to species other than iron were measured to compute differential abundances of 20 chemical elements in the $\psi^{1}$ Draconis stars. The line list and adopted atomic data, including hyperfine structure parameters when available, are from Ramírez et al. (2009, 2011). Oxygen abundances were inferred using the $\mathrm{O}_{\text {I }}$ triplet lines at $777 \mathrm{~nm}$, corrected for non-LTE effects using the grid by Ramírez et al. (2007).

The relative elemental abundances we measured are plotted in Figure 23 as a function of the elements' $50 \%$ condensation temperatures, as computed by Lodders (2003) for a solar composition gas. Note that this "A-B" difference in chemical abundances was obtained when $\psi^{1}$ Dra A was directly analyzed with respect to $\psi^{1}$ Dra B in a strict line-by-line differential manner. The error bars are significantly smaller compared to the case in which the elemental abundances are first determined with respect to the Sun and then subtracted. This is a consequence of reducing the systematic errors of the analysis by avoiding a reference that is very dissimilar to either one of the $\psi^{1}$ Draconis stars.

Figure 23 shows that $\psi^{1}$ Dra A is metal-poor relative to $\psi^{1}$ Dra B. On average, the metallicity difference is $-0.09 \pm 0.04$ dex. We do not detect a statistically significant correlation with the condensation temperature, but this is likely due to the relatively large errors in the abundance differences. In the Meléndez et al. and Ramírez et al. works the precision of relative abundances is of order $0.01 \mathrm{dex}$. In our case those errors are about 0.04 dex instead. Thus, we cannot rule out possible trends based on our data.

One may be tempted to attribute the elemental abundance discrepancy shown in Figure 23 to uncertain stellar parameters. The derived chemical abundances are most affected by the adopted $T_{\text {eff }}$ values, and we have shown above that those of $\psi^{1}$ Dra B are reliable. Thus, we can explore this potential systematic error by simply calculating the relative abundances for different $T_{\text {eff }}$ values for $\psi^{1}$ Dra A and keeping everything else constant. Increasing the $T_{\text {eff }}$ of $\psi^{1}$ Dra A by $200 \mathrm{~K}$ would make the average elemental abundance difference nearly zero, but only for refractory elements $\left(T_{\text {cond }} \gtrsim 1000 \mathrm{~K}\right)$. The abundances of $\mathrm{C}$ and $\mathrm{O}$ in this case would differ by about -0.2 dex. On the other hand, decreasing the $T_{\text {eff }}$ of $\psi^{1}$ Dra A by $200 \mathrm{~K}$ would make the $\mathrm{C}$ and $\mathrm{O}$ abundance difference nearly zero, but then the refractories would differ by about -0.2 dex. In both cases, we note that the element-to-element scatter and the line-to-line relative abundance scatter for individual elements increase relative to the case when our derived $T_{\text {eff }}$ value is adopted instead. In other words, the elemental abundance differences are more internally consistent for our derived parameters, suggesting that the hotter or cooler temperatures of $\psi^{1}$ Dra A are not realistic (within our modeling assumptions, of course). Thus, it is not possible to reconcile the chemical abundance difference between $\psi^{1}$ Dra A and B by assuming that the $T_{\text {eff }}$ of the former is either underestimated or overestimated. The spectral contamination of $\psi^{1}$ Dra A cannot explain the observed abundance difference either. Since only $1 \%$ of the flux is from the low-mass stellar companion, the equivalent widths and abundances derived could have been underestimated by $1 \%$ at most. This corresponds to less than about 0.005 dex in $[\mathrm{X} / \mathrm{H}]$. We are led to conclude that the offset seen in Figure 23 is real.

\subsubsection{Does $\psi^{1}$ Dra A Have $\delta$ Scuti Abundances?}

The chemical composition of $\delta$ Scuti stars may be peculiar. The prototype star of this class shows a severe enhancement, up to about $1.0 \mathrm{dex}$, in the abundances of elements with atomic number above 30 (Yushchenko et al. 2005). A very similar abundance pattern is observed in $\rho$ Puppis, a very bright $\delta$ Scuti star, as shown by Gopka et al. (2007). Note, however, that both $\delta$ Scuti and $\rho$ Puppis are about $500 \mathrm{~K}$ warmer than $\psi^{1}$ Dra A

A $\delta$ Scuti star with known detailed chemical abundances that is more similar in stellar parameters to $\psi^{1}$ Dra A is CP Boo (Galeev et al. 2012). This star is about $200 \mathrm{~K}$ cooler than $\psi^{1}$ Dra $\mathrm{A}$ and a bit more metal-rich $([\mathrm{Fe} / \mathrm{H}]=+0.16)$. The abundances measured by Galeev et al. also reveal enhancements of the heavy metals, but not as dramatic as in $\delta$ Scuti and $\rho$ Puppis. On average, the abundances of elements with atomic number above 30 are higher by about 0.3 dex. Such a level of enhancement would be easily detected in our spectra. Figure 23 shows that at least the abundances of $\mathrm{Zn} \quad(\mathrm{Z}=30$, $\mathrm{Tc}=726 \mathrm{~K}), \quad \mathrm{Y} \quad(\mathrm{Z}=39, \quad \mathrm{Tc}=1659 \mathrm{~K}), \quad \mathrm{Zr} \quad(\mathrm{Z}=40$, $\mathrm{Tc}=1741 \mathrm{~K})$, and $\mathrm{Ba}(\mathrm{Z}=56, \quad \mathrm{Tc}=1455 \mathrm{~K})$ are not enhanced in $\psi^{1}$ Dra A relative to $\psi^{1}$ Dra B. They are also not enhanced when the abundances are measured relative to the solar abundances.

To provide further evidence for this finding, we measured the abundances of $\mathrm{Nd}(\mathrm{Z}=60, \mathrm{Tc}=1594 \mathrm{~K})$ and $\mathrm{Eu}(\mathrm{Z}=63$, $\mathrm{Tc}=1347 \mathrm{~K})$ using spectrum synthesis following the procedure described in Ramírez et al. (2011). For both of these species we found an A-B difference of $-0.10+/-0.06$ dex. In other words, the $\mathrm{Nd}$ and $\mathrm{Eu}$ abundances of $\psi^{1}$ Dra A relative to $\psi^{1}$ Dra B fit perfectly the pattern seen in Figure 23. In particular, they are also not enhanced in the former. An enhancement of $1.0 \mathrm{dex}$, as in $\delta$ Scuti or $\rho$ Puppis, or even the mild enhancement of 0.3 dex seen in CP Boo would have been trivial to detect in our analysis. In fact, in that case some points would be found out of the chart in Figure 23. 
Although the abundance pattern of $\psi^{1}$ Dra A does not look like that of some prototypical $\delta$ Scuti stars, it should be noted that these peculiarities may not correlate perfectly with the stars' pulsation characteristics. In fact, Fossati et al. (2008) argue that $\delta$ Scuti stars have abundance patterns that are indistinguishable from a sample of normal A- and F-type stars. Thus, the nonenhancement of heavy metal abundances that we find for $\psi^{1}$ Dra A does not necessarily rule it out as a candidate for a star of the $\delta$ Scuti class.

\subsubsection{Possible Interpretations}

In the 16 Cygni system, the secondary hosts a gas giant planet, whereas the primary has not yet shown evidence of substellar-mass companions. Ramírez et al. (2011) found that $16 \mathrm{Cyg} \mathrm{B}$ is slightly metal-poor relative to $16 \mathrm{Cyg} \mathrm{A}$ and explained this observation as a signature of planet formation. Briefly, they suggested that the missing metals of 16 Cyg B are currently located inside its planet. Considering that hypothesis, possible explanations for our results regarding the $\psi^{1}$ Draconis system include the following:

1. The 16 Cygni planet signature hypothesis is incorrect because in $\psi^{1}$ Draconis the secondary, which is a gas giant planet host, is actually more metal-rich than the primary, which does not show evidence of hosting planets in our RV data. Metals should have been taken away from the planet-host star $\psi^{1} \operatorname{DraB}$ and that star should be metal-poor relative to $\psi^{1}$ Dra A, which is the opposite of what we observe. In this case, the cause of the observed abundance differences seen in both 16 Cygni and $\psi^{1}$ Draconis remains unknown.

2. Planet-like material and perhaps even fully formed planets were once present in orbit around $\psi^{1}$ Dra A, with a total mass greater than that of $\psi^{1}$ Dra B's planet or planets combined. However, the stellar companion $\psi^{1}$ Dra $\mathrm{C}$ made the planetary environment unstable, ejecting all of the planet material away from $\psi^{1}$ Dra A. In this scenario, the outer layers of $\psi^{1}$ Dra A would have accreted metal-poor gas during the planet-formation stage. The metals missing from $\psi^{1}$ Dra A would have been locked up in the material that was ejected later. The late ejection of that material is required to explain our nondetection of planets around $\psi^{1}$ Dra A. Since $\psi^{1}$ Dra B has a planet (or two), its atmosphere is also depleted in metals relative to the initial metallicity of the system, but the metal depletion suffered by $\psi^{1}$ Dra A was greater. The latter would be easily explained by a larger total mass of planet-like material, but it could also be in part due to the thinner convective envelope of this warmer star, which did not dilute the chemical signature as much as $\psi^{1}$ Dra B.

3. $\psi^{1}$ Dra A never formed planets owing to the influence of its low-mass stellar companion. On the other hand, $\psi^{1}$ Dra B formed much more planet-like material than seen today in the planet or planets that we have detected. A fraction of this material, that which is not in the planet(s) detected by us, was accreted into the star at a later stage, increasing the metallicity of its atmosphere. The amount of planet material accreted that is necessary to explain our observations had to have been larger than the total mass of the planet or planets detected. This is because the formation of those planets implies that metals were already taken away from the star, and this needs to be
Table 11

Differential Radial Velocity and Ca H \& K Observations for HD 10086 (Sample)

\begin{tabular}{lcrccc}
\hline \hline Spectrum & BJD & $\begin{array}{c}\mathrm{dRV} \\
\left(\mathrm{m} \mathrm{s}^{-1}\right)\end{array}$ & $\begin{array}{c}\text { Uncertainty } \\
\left(\mathrm{m} \mathrm{s}^{-1}\right)\end{array}$ & $S_{\mathrm{HK}}$ & Uncertainty \\
\hline 1 & $2,451,152.7300$ & 7.0 & 7.2 & 0.312 & 0.020 \\
2 & $2,451,213.6509$ & -0.8 & 5.4 & 0.302 & 0.018 \\
3 & $2,451,240.6034$ & 37.4 & 10.9 & 0.299 & 0.017 \\
4 & $2,451,452.8818$ & 38.6 & 6.0 & 0.335 & 0.020 \\
5 & $2,451,503.7088$ & 21.5 & 7.1 & 0.327 & 0.021 \\
6 & $2,451,530.7712$ & 4.9 & 6.2 & 0.290 & 0.019 \\
7 & $2,451,558.6106$ & 20.5 & 6.6 & 0.304 & 0.020 \\
8 & $2,451,775.9029$ & -2.8 & 5.7 & 0.273 & 0.020 \\
9 & $\ldots$ & $\ldots$ & $\ldots$ & $\cdots$ & $\cdots$ \\
\hline
\end{tabular}

(This table is available in its entirety in machine-readable form.)

first compensated in order to result in a stellar atmosphere that is more metal-rich than the birth cloud of the system. In this scenario, the metallicity of $\psi^{1}$ Dra $\mathrm{A}$ is that of the gas cloud from which both stars formed, whereas $\psi^{1}$ Dra B's atmosphere became metal-rich at a later stage.

4. Planets did also form around $\psi^{1}$ Dra A, but we did not detect them because the $\delta$ Scuti pulsations and spectral contamination from $\psi^{1}$ Dra C lead to the observed large $\mathrm{RV}$ jitter that prevents the detection of the RV signature of any planet around this star. Another way the planets could avoid detection by the RV method is if the angle between the planetary orbital planes and the sky is small.

Finally, we note that the potential post-main-sequence nature of $\psi^{1}$ Dra A could have allowed dredge-up to occur in that star. However, this mechanism is expected to enhance the metallicity of the stellar convection zone and photosphere, while the effect that we observe is that of a depletion of metals in $\psi^{1}$ Dra A. Thus, dredge-up can also be ruled out as a possibility to explain the abundance offset seen in Figure 23.

\section{TWO COLD JUPITER "FALSE ALARMS" RELATED TO STELLAR ACTIVITY}

\subsection{HD 10086}

We have obtained 84 RV measurements of HD 10086 over approximately $16 \mathrm{yr}$, as listed in Table 11 . The RVs have an rms of $13.1 \mathrm{~m} \mathrm{~s}^{-1}$ with a mean uncertainty of $6.3 \mathrm{~m} \mathrm{~s}^{-1}$, indicating the potential presence of a periodic signal. The periodogram of the velocities (Figure 24) shows a broad and significant peak centered at 2800 days. This signal may be modeled as a circular Keplerian orbit with period 2800 days and a semiamplitude $K=11 \mathrm{~m} \mathrm{~s}^{-1}$, which would correspond to a planet with a minimum mass $M \sin i=0.74 M_{\text {Jup }}$ at $a=3.9$ AU. Incidently, HD 10086 was also included in the Lick Observatory RV survey (Fischer et al. 2014). The 40 Lick RVs have an rms of $18.6 \mathrm{~m} \mathrm{~s}^{-1}$ and a mean uncertainty of $3.6 \mathrm{~m} \mathrm{~s}^{-1}$. This independently confirms the apparent RV variability of this star.

However, in our analysis of stellar activity indicators for HD 10086, we see a similar 2800-day peak in the periodogram of the $S_{\mathrm{HK}} \mathrm{Ca} \mathrm{H} \& \mathrm{~K}$ index, suggesting that the RV modulation may reflect a stellar activity cycle rather than a giant planet. Considering RV as a function of $S_{\mathrm{HK}}$ (Figure 25, top panel) confirms this hypothesis. The RVs of HD 10086 are very strongly correlated with $S_{\mathrm{HK}}$; a Pearson correlation test yields a 


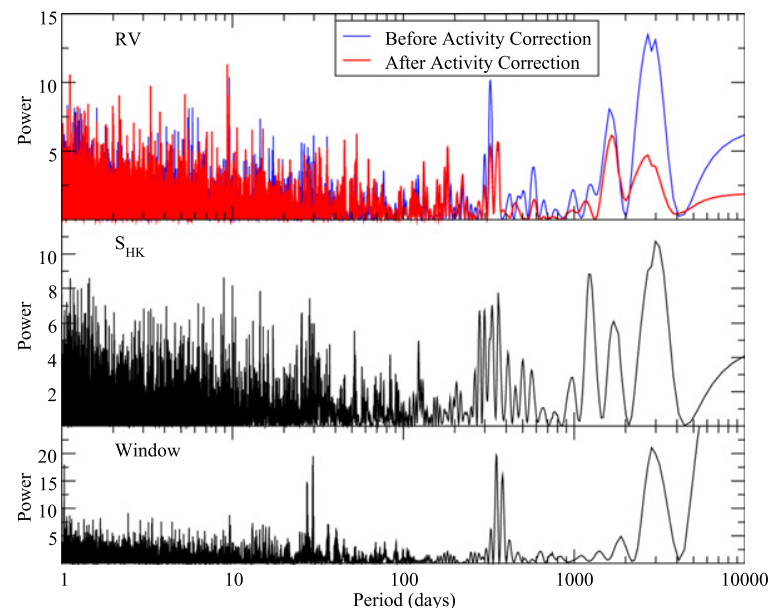

Figure 24. Generalized Lomb-Scargle periodograms of our RV data for HD 10086 before (blue) and after (red) correcting for stellar activity, along with the corresponding periodogram of $S_{\mathrm{HK}}$.
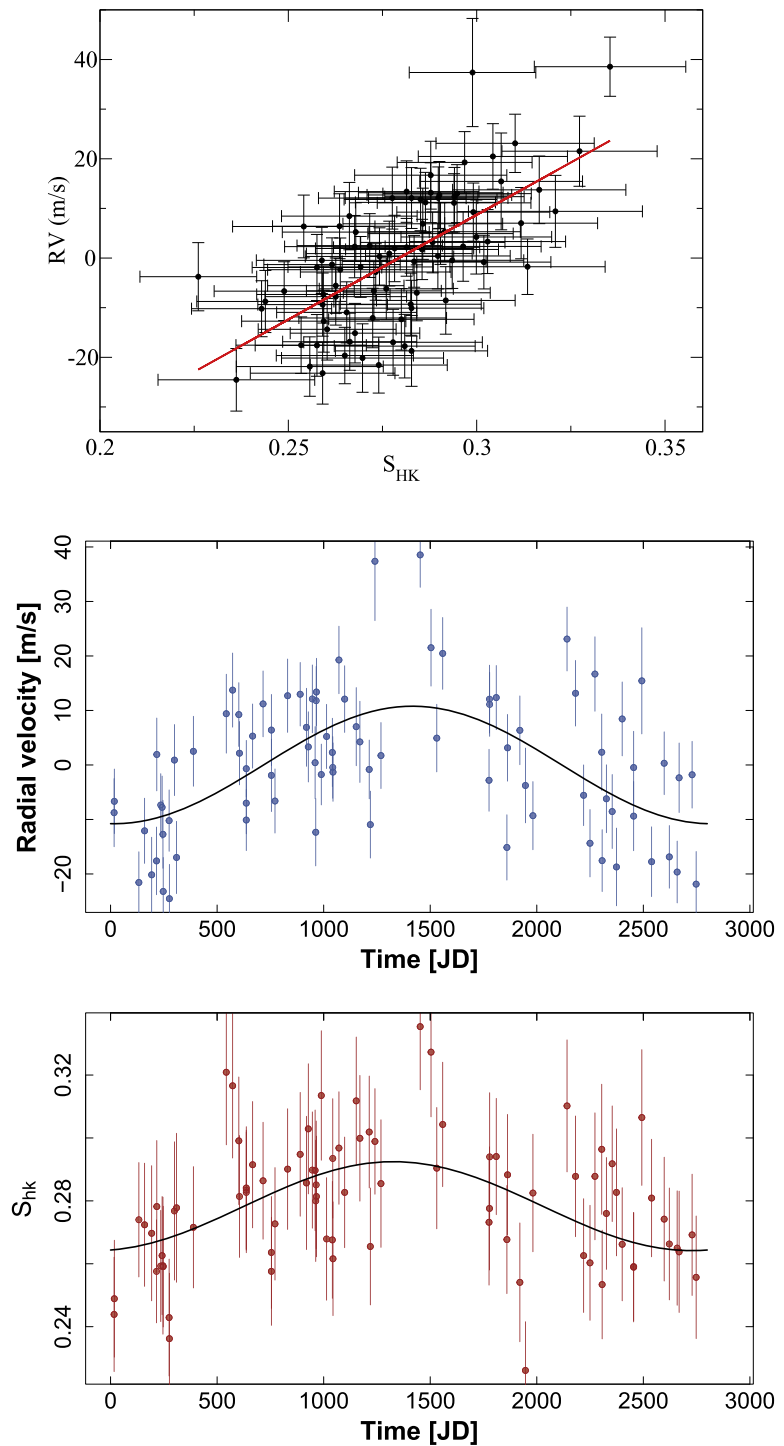

Figure 25. Top panel: RV for HD 10086 as a function of $S_{\mathrm{HK}}$ at the time of each observation. The linear least-squares fit to the relation is given as a solid red line. Middle and bottom panels: RV and $S_{\mathrm{HK}}$, respectively, folded to the 2800-day period of the stellar activity cycle. Sinusoidal models to each data set are shown as solid black curves.
Table 12

Differential Radial Velocity and Ca H \& K Observations for $\beta$ Virginis (Sample)

\begin{tabular}{lcrccc}
\hline \hline Spectrum & BJD & $\begin{array}{c}\mathrm{dRV} \\
\left(\mathrm{m} \mathrm{s}^{-1}\right)\end{array}$ & $\begin{array}{c}\text { Uncertainty } \\
\left(\mathrm{m} \mathrm{s}^{-1}\right)\end{array}$ & $S_{\mathrm{HK}}$ & Uncertainty \\
\hline 1 & $2,451,009.6241$ & 10.9 & 2.4 & 0.158 & 0.014 \\
2 & $2,451,153.9622$ & 0.7 & 6.0 & 0.164 & 0.020 \\
3 & $2,451,213.0360$ & -4.4 & 2.5 & 0.159 & 0.020 \\
4 & $2,451,241.8748$ & -11.3 & 3.2 & 0.176 & 0.020 \\
5 & $2,451,274.7687$ & 3.1 & 4.2 & 0.179 & 0.019 \\
6 & $2,451,326.7453$ & 1.6 & 3.1 & 0.168 & 0.017 \\
7 & $2,451,358.6645$ & 8.2 & 2.0 & 0.162 & 0.016 \\
8 & $2,451,504.0169$ & 2.0 & 2.2 & 0.166 & 0.023 \\
9 & $\ldots$ & $\cdots$ & $\cdots$ & $\cdots$ & $\cdots$ \\
\hline
\end{tabular}

(This table is available in its entirety in machine-readable form.)

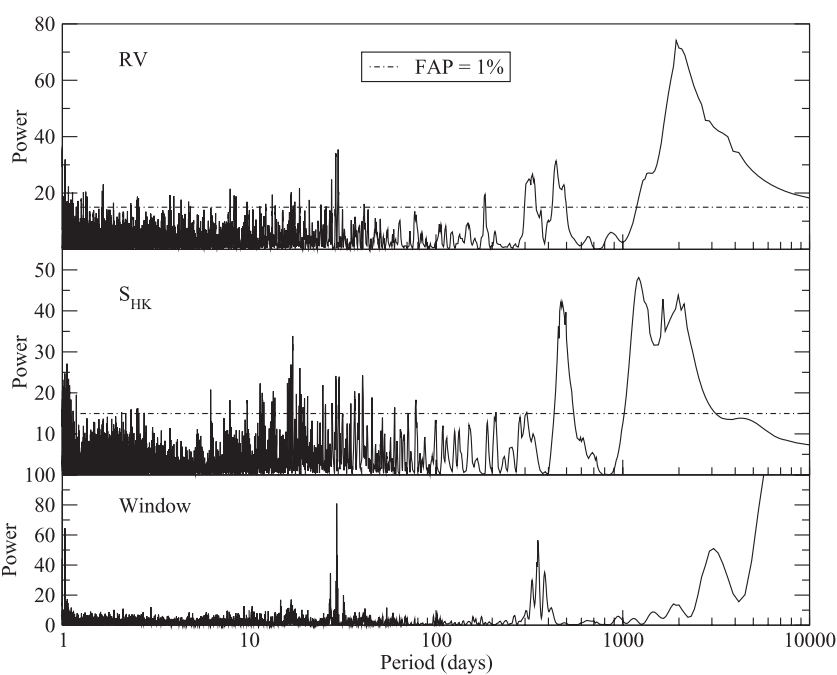

Figure 26. Generalized Lomb-Scargle periodograms of RV and $S_{\mathrm{HK}}$ for $\beta$ Virginis. The threshold for an FAP of $1 \%$ is shown as a dot-dashed line.

correlation coefficient $r=0.66$, which, for a sample size $N=84$, indicates a probability of $P<10^{-12}$ that we would observe such a correlation if $\mathrm{RV}$ and activity were uncorrelated.

Given the tight correlation between $\mathrm{RV}$ and $\mathrm{Ca} \mathrm{H}$ \& $\mathrm{K}$ emission for this star, we attempted to perform a simple stellar activity correction by fitting and removing a linear least-squares model for RV versus $S_{\mathrm{HK}}$. We find a linear fit of $v_{r}=-120(15)+420(50) \times S_{\mathrm{HK}}$. Upon subtracting this model from the RVs, we see from the activity-corrected periodogram that the 2800-day signal is almost completely eliminated, providing final confirmation that this signal is caused by Doppler shifts associated with a $7.7 \mathrm{yr}$ activity cycle. We show both RV and $S_{\mathrm{HK}}$ folded to the period of this cycle in Figure 25 . We see no statistically significant additional signals in $\mathrm{RV}$ and conclude that we have not discovered any exoplanets around this star to date.

\section{2. $\beta$ Virginis}

We have observed $\beta$ Virginis (hereafter $\beta$ Vir) for approximately $16 \mathrm{yr}$, obtaining a total of $311 \mathrm{RV}$ measurements, as listed in Table 12. These velocities have an rms of $9.0 \mathrm{~m} \mathrm{~s}^{-1}$ with a mean uncertainty of just $3.7 \mathrm{~m} \mathrm{~s}^{-1}$. In Figure 26, we show the Lomb-Scargle periodogram of the RVs, which includes a broad, highly significant peak at 

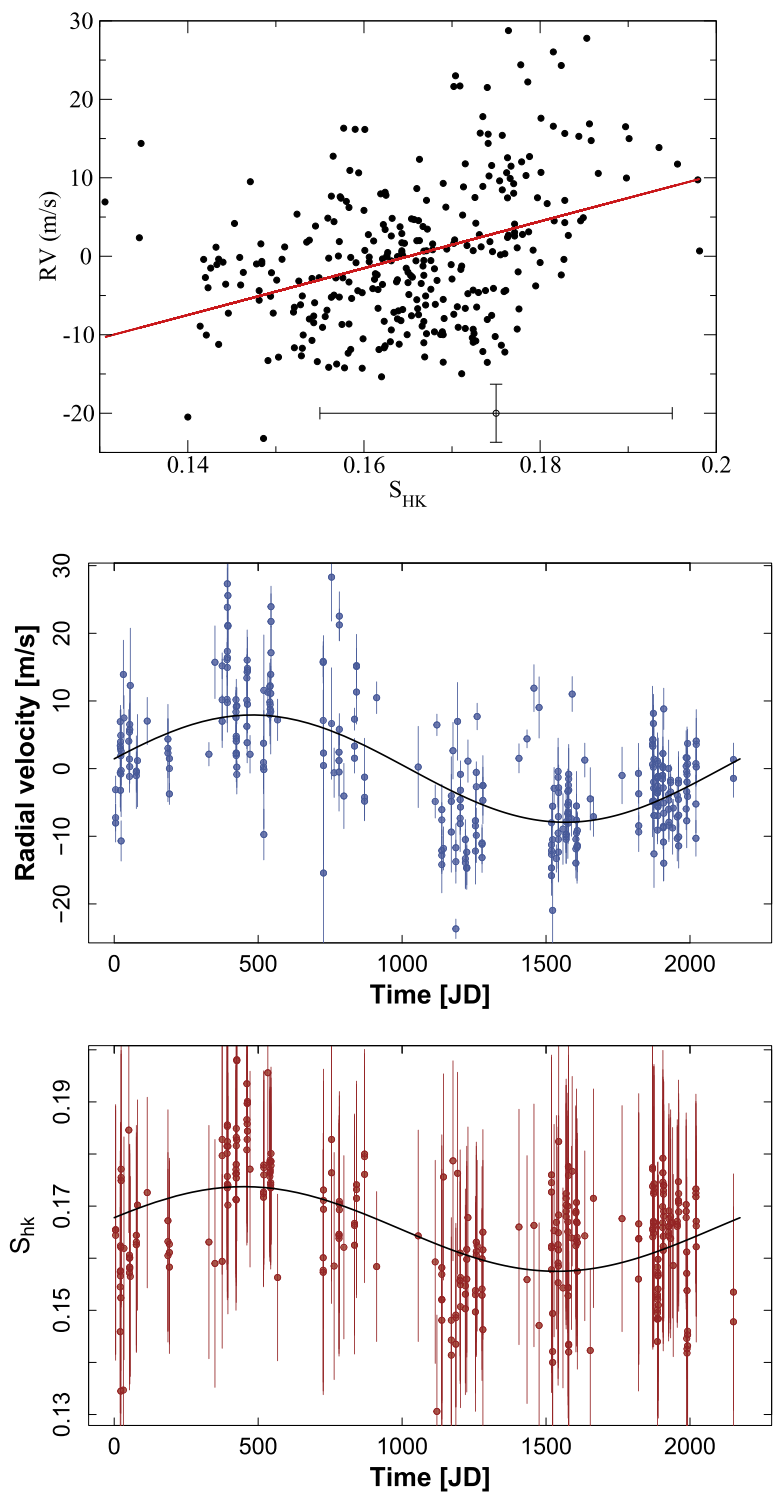

Figure 27. Top panel: RV as a function of $S_{\mathrm{HK}}$ for $\beta$ Virginis. The linear leastsquares fit to the data is shown as a solid red line. For the sake of visibility we do not show error bars on the individual points, but indicate the mean $1 \sigma$ uncertainty on each variable. Middle and bottom panels: $\mathrm{RV}$ and $S_{\mathrm{HK}}$, respectively, folded to the 2200-day period of the stellar activity cycle. Sinusoidal models to each data set are shown as solid black curves.

approximately 2000 days. The best Keplerian model to the data produces an eccentric $(e=0.26)$ orbit with $P=2040$ days and $K=9 \mathrm{~m} \mathrm{~s}^{-1}$

If the RV modulation is indeed produced by an exoplanet, this Keplerian model corresponds to a gas giant orbiting at $a=3.5 \mathrm{AU}$ with minimum mass of $M \sin i=0.65 M_{\mathrm{Jup}}$. As with HD 10086, though, the $\mathrm{Ca} \mathrm{H} \& \mathrm{~K}$ emission of $\beta$ Vir suggests that the observed signal is not associated with a planet. We include the periodogram of $S_{\mathrm{HK}}$ in Figure 26, which also includes a very broad peak between 1000 and 2500 days. RV as a function of $S_{\mathrm{HK}}$ (Figure 27) again shows a highly significant correlation; we compute a Pearson correlation coefficient $r=0.39$ and a $P$-value of $2 \times 10^{-12}$. We therefore suspect that the periodicity observed for $\beta$ Vir is also a stellar activity cycle that mimics a Doppler shift, such as would be expected for a Jupiter-analog planet.
A number of features of our data set for $\beta$ Vir prevent the application of a simple stellar activity correction analogous to the one we performed for HD 10086. First, the weaker calcium emission (mean $S_{\mathrm{HK}}=0.17$, versus 0.28 for HD 10086) leads to lower $\mathrm{S} / \mathrm{N}$ in the $\mathrm{Ca} \mathrm{H} \& \mathrm{~K}$ measurements. Furthermore, our RVs show significant short-term scatter $\left(5.1 \mathrm{~m} \mathrm{~s}^{-1}\right.$ over the 2013 observing season) and possibly a long-term linear acceleration in addition to the activity-induced periodicity. Finally, the "eccentricity" of the RV signal created by the activity cycle suggests that the activity signal may be nonsinusoidal, and the RV-activity relationship may therefore not be linear. These factors make it especially difficult to fit and subtract a simple activity-RV dependence, and we therefore do not attempt a stellar activity correction for $\beta$ Vir. The matching periodicities in $\mathrm{RV}$ and $\mathrm{Ca} \mathrm{H} \& \mathrm{~K}$ and the correlation between $\mathrm{RV}$ and $S_{\mathrm{HK}}$ lead us to conclude that the observed signal is due to a stellar activity cycle, but the evaluation of any additional (possibly planetary) signals in the velocities must be postponed pending a more sophisticated stellar activity analysis, which is beyond the scope of this paper.

\section{DISCUSSION AND CONCLUSION}

We present two cases (HD 95782 and $\psi^{1}$ Dra B) for gas giant planets at large orbital separations, in the Jupiter-analog range. Owing to the very long time baseline of over a decade or more, the RV discoveries of such planets are still relatively rare. Long-term precise RV surveys, like the McDonald Observatory planet search, still represent the current best capability to find these planets. These planets cannot be found by Kepler, nor by K2, nor by TESS (Ricker et al. 2015), owing to the short time span of monitoring, coupled with a very low transit probability of planets at $5 \mathrm{AU}$. And, despite that the best direct imaging instruments like Gemini Planet Imager (GPI, Macintosh et al. 2014) and SPHERE (Beuzit et al. 2008) reach the small inner working angles for nearby stars, the low luminosities of mature, old Jupiters make them virtually undetectable, even for these instruments. Discoveries of giant planet candidates like 51 Eri b (Macintosh et al. 2015) at very young ages of $\sim 20 \mathrm{Myr}$ and separations of $a>10 \mathrm{AU}$ will eventually allow us to find a complete picture for these type of planets in time and orbital separation space.

Another advantage of long-term RV surveys is the fact that we can use the RV data for all stars, where we do not detect a planet, to set tight constraints for the presence of these gas giants. These will allow us to determine the occurrence rate of Jupiter analogs, and even of solar-system-type architectures with two gas giants.

The possibly crucial role of Jupiter, as well as of Saturn, for the formation of the terrestrial planets in our solar system has been highlighted recently by Batygin \& Laughlin (2015). These authors present a model, within the context of the "Grand Tack" model (Walsh et al. 2011), that explains why we do not have super-Earths in the inner solar system, like the numerous Kepler systems. In their model, the migration of Jupiter (which is halted and reversed by Saturn's dynamical evolution) depletes the interior planetesimal disk, possibly driving all existing short-period super-Earths into the Sun. The low-mass terrestrial planets then subsequently formed in this depleted disk in the inner region of our solar system. This model would therefore predict that planetary systems similar to ours can only form with at least two gas giants that end up at large separations after the early phase of migration has finished. The 
search for long-period gas giants therefore gains importance also in the search for Earth-like planets. The $\psi^{1}$ Dra B planetary system could be an excellent candidate for a system with a planetary architecture very similar to our own, with two gas giants at large multi-AU separations, which possibly also helped the formation of lower-mass rocky planets in the inner few AUs.

The other two stars, HD 10086 and $\beta$ Vir, are stark reminders that stellar activity can mimic also the presence of Jupiter analogs. Long-term magnetic cycles can present themselves as slow RV modulations very similar to a Jupiter. Given that our Sun's magnetic field cycle is comparable to Jupiter's orbital period, we need to develop techniques that can correct for these effects and make planet detection possible, even in the presence of a stellar activity cycle. Our first approach to correlate the chromospheric emission in the $\mathrm{Ca}$ II $\mathrm{H}$ \& $\mathrm{K}$ lines with the $\mathrm{RV}$ signal works in a simple case like HD 10086. The need for a more sophisticated model is obvious in the case of $\beta$ Vir. The relatively large RV amplitudes of the activity signal of several $\mathrm{m} \mathrm{s}^{-1}$ are somewhat unexpected, especially for the relatively inactive $\operatorname{star} \beta$ Vir. However, the SARG binary planet survey also found an activity cycle of the star HD $200466 \mathrm{~A}$, which produces an RV signal with a semiamplitude of $\approx 20 \mathrm{~m} \mathrm{~s}^{-1}$ (Carolo et al. 2014). It is thus clear that in our search for Jupiter analogs, we need to expect activity-induced RV signals that can mimic even massive gas giants. Fortunately, all our spectra from the Tull instrument contain the $\mathrm{Ca}$ II $\mathrm{H} \& \mathrm{~K}$ lines and permit us an immediate test for possible activity cycles. The very long duration McDonald Observatory planet search at the $2.7 \mathrm{~m} \mathrm{HJST/Tull} \mathrm{will}$ therefore provide a unique data set for its entire sample of over 200 stars to find planetary systems similar to our own.

This work has been made possible through the National Science Foundation (Astrophysics grant AST-1313075) and various NASA grants over the years. We are grateful for their generous support. We also thank the McDonald Observatory Time Allocation committee for its continuing support of this program.

This research has made use of the SIMBAD database, operated at CDS, Strasbourg, France.

We would also like to thank all the observers who have helped gather data over the years for the $2.7 \mathrm{~m} \mathrm{RV}$ planet search program at McDonald Observatory, including Diane Paulson, Stuart I. Barnes, Candice Gray, and Anita Cochran.

Finally, we would like to thank the anonymous referee; her/ his comments helped to improve this paper.

Some of the data presented herein were obtained at the W.M. Keck Observatory, which is operated as a scientific partnership among the California Institute of Technology, the University of California, and the National Aeronautics and Space Administration. The Observatory was made possible by the generous financial support of the W.M. Keck Foundation. The authors wish to recognize and acknowledge the very significant cultural role and reverence that the summit of Mauna Kea has always had within the indigenous Hawaiian community. We are most fortunate to have the opportunity to conduct observations from this mountain.

Facilities: Gemini Observatory (DSSI), McDonald Observatory (Tull Coudé Spectropgraph), Keck-I Observatory (HIRES).

\section{REFERENCES}

Andrae, R., Schulze-Hartung, T., \& Melchior, P. 2010, arXiv:1012.3754 Baliunas, S. L., Donahue, R. A., Soon, W. H., et al. 1995, ApJ, 438, 269 Balona, L. A., Daszyńska-Daszkiewicz, J., \& Pamyatnykh, A. A. 2015, MNRAS, 452, 3073

Batygin, K., \& Laughlin, G. 2015, PNAS, 112, 4214

Bedell, M., Meléndez, J., Bean, J. L., et al. 2015, A\&A, 581, A34

Beuzit, J.-L., Feldt, M., Dohlen, K., et al. 2008, Proc. SPIE, 7014, 18

Borucki, W. J., Koch, D., Basri, G., et al. 2010, Sci, 327, 977

Brugamyer, E., Dodson-Robinson, S. E., Cochran, W. D., \& Sneden, C. 2011, ApJ, 738, 97

Carolo, E., Desidera, S., Gratton, R., et al. 2014, A\&A, 567, 48

Casagrande, L., Ramírez, I., Meléndez, J., Bessell, M., \& Asplund, M. 2010, A\&A, 512, 54

Chambers, J. E. 1999, MNRAS, 304, 793

Chambers, J. E., Wetherill, G. W., \& Boss, A. P. 1996, Icar, 119, 261

Cochran, W. D., \& Hatzes, A. P. 1993, in ASP Conf. Ser. 36, Planets around Pulsars, ed. J. A. Phillips, J. E. Thorsett, \& S. R. Kulkarni (San Fransisco, CA: ASP), 267

Cochran, W. D., Hatzes, A. P., Butler, R. P., \& Marcy, G. W. 1997, ApJ, 483, 457

Endl, M., Kürster, M., \& Els, S. 2000, A\&A, 362, 585

ESA 1997, yCat, 1239

Fischer, D. A., Marcy, G. W., \& Spronck, J. F. P. 2014, ApJS, 210, 5

Ford, E. B. 2005, AJ, 129, 1706

Ford, E. B. 2006, ApJ, 642, 505

Fossati, L., Kolenberg, K., Reegen, P., \& Weiss, W. 2008, A\&A, 485, 257

Fressin, F., Torres, G., Charbonneau, D., et al. 2013, ApJ, 766, 81

Galeev, A. I., Ivanova, D. V., Shimansky, V. V., \& Bikmaev, I. F. 2012, ARep, 56,850

González Hernández, J. I., Delgado-Mena, E., Sousa, S. G., et al. 2013, A\&A, 552, A6

González Hernández, J. I., Israelian, G., Santos, N. C., et al. 2010, ApJ, 720,1592

Gopka, V., Yushchenko, A., Kim, C., et al. 2007, in ASP Conf. Ser. 362, The Seventh Pacific Rim Conf. on Stellar Astrophysics, ed. Y. W. Kang et al. (San Francisco, CA: ASP), 249

Gregory, P. C. 2011, MNRAS, 415, 2523

Gullikson, K., Endl, M., Cochran, W. D., \& MacQueen, P. J. 2015, ApJ, 815,62

Hatzes, A. P., Cochran, W. D., Endl, M., et al. 2003, ApJ, 599, 1383

Hatzes, A. P., Cochran, W. D., \& Johns-Krull, C. M. 1997, ApJ, 478, 374

Horch, E. P., Gomez, S., Sherry, W. H., et al. 2011, AJ, 141, 45

Horch, E. P., Howell, S. B., Everett, M. E., \& Ciardi, D. R. 2012, AJ, 144, 165

Horch, E. P., Veillette, D. R., Baena Gallé, R., et al. 2009, AJ, 137, 5057

Horner, J., Hinse, T. C., Wittenmyer, R. A., Marshall, J. P., \& Tinney, C. G. 2012a, MNRAS, 427, 2812

Horner, J., \& Jones, B. W. 2008, IJAsB, 7, 251

Horner, J., \& Jones, B. W. 2010, IJAsB, 9, 273

Horner, J., \& Jones, B. W. 2012, IJAsB, 11, 147

Horner, J., Jones, B. W., \& Chambers, J. 2010, IJAsB, 9, 1

Horner, J., Wittenmyer, R. A., Hinse, T. C., \& Tinney, C. G. 2012b, MNRAS, 425,749

Howard, A. W., Johnson, J. A., Marcy, G. W., et al. 2010, ApJ, 721, 1467

Howard, A. W., Marcy, G. W., Bryson, S. T., et al. 2012, ApJS, 201, 15

Howard, A. W., Marcy, G. W., Fischer, D., et al. 2014, ApJ, 794, 51

Kim, Y., Demarque, P., Yi, S., \& Alexander, D. R. 2002, ApJS, 143, 499

Kharchenko, N. V., \& Roeser, S. 2009, yCat, 1280

Koen, C. 2006, MNRAS, 371, 1390

Kürster, M., Schmitt, J. H. M. M., Cutispoto, G., \& Dennerl, K. 1997, A\&A, 320,831

Kurucz, R. L. 1993, yCat, 6039

Lodders, K. 2003, ApJ, 591, 1220

Macintosh, B., Anthony, A., Atwood, J., et al. 2014, Proc. SPIE, 9148, 0

Macintosh, B., Graham, J. R., Barman, T., et al. 2015, Sci, 350, 64

Marcy, G. W., Butler, R. P., Vogt, S. S., et al. 2005, ApJ, 619, 570

Marmier, M., Ségransan, D., Udry, S., et al. 2013, A\&A, 551, 90

Meléndez, J., Asplund, M., Gustafsson, B., \& Yong, D. 2009, ApJL, 704, L66

Meschiari, S., Wolf, A. S., Rivera, E., et al. 2009, PASP, 121, 1016

Moutou, C., Lo Curto, G., Mayor, M., et al. 2015, A\&A, 576, 48

Paulson, D. B., Saar, S. H., Cochran, W. D., \& Hatzes, A. P. 2002, AJ, 124,572

Petigura, E. A., Howard, A. W., \& Marcy, G. W. 2013, PNAS, 110, 19273

Ramírez, I., Allende Prieto, C., \& Lambert, D. L. 2007, A\&A, 465, 271 
Ramírez, I., Allende Prieto, C., \& Lambert, D. L. 2013, ApJ, 764, 78

Ramírez, I., Meléndez, J., \& Asplund, M. 2009, A\&A, 508, L17

Ramírez, I., Meléndez, J., Bean, J., et al. 2014, A\&A, 572, 48

Ramírez, I., Meléndez, J., Cornejo, D., Roederer, I. U., \& Fish, J. R. 2011, ApJ, 740,76

Raymond, S. N. 2006, ApJ, 643, 131

Ricker, G. R., Winn, J. N., Vanderspek, R., et al. 2015, JATIS, 1, 014003

Robertson, P., Endl, M., Cochran, W. D., et al. 2012a, ApJ, 749, 39

Robertson, P., Horner, J., Wittenmyer, R. A., et al. 2012b, ApJ, 754, 50

Schuler, S. C., Flateau, D., Cunha, K., et al. 2011, ApJ, 732, 55

Soderblom, D. R., Duncan, D. K., \& Johnson, D. R. H. 1991, ApJ, 375, 722

Toyota, E., Itoh, Y., Ishiguma, S., et al. 2009, PASJ, 61, 19

Tucci Maia, M., Meléndez, J., \& Ramírez, I. 2014, ApJL, 790, L25
Tull, R. G., MacQueen, P. J., Sneden, C., \& Lambert, D. L. 1995, PASP, 107,251

Vogt, S. S., Allen, S. L., Bigelow, B. C., et al. 1994, Proc. SPIE, 2198, 362

Walsh, K. J., Morbidelli, A., Raymond, S. N., O’Brien, D. P., \& Mandell, A. M. 2011, Natur, 475, 206

Wittenmyer, R. A., Horner, J., \& Tinney, C. G. 2012a, ApJ, 761, 165

Wittenmyer, R. A., Horner, J., Tinney, C. G., et al. 2014a, ApJ, 783, 103

Wittenmyer, R. A., Horner, J., Tuomi, M., et al. 2012b, ApJ, 753, 169

Wittenmyer, R. A., Tan, X., Lee, M. H., et al. 2014b, ApJ, 780, 140

Wittenmyer, R. A., Tinney, C. G., Butler, R. P., et al. 2011, ApJ, 738, 81

Yi, S., Demarque, P., Kim, Y., et al. 2001, ApJS, 136, 417

Yushchenko, A., Gopka, V., Kim, C., et al. 2005, MNRAS, 359, 865

Zechmeister, M., \& Kürster, M. 2009, A\&A, 496, 577 